\title{
STABILITY AND ERROR ANALYSIS OF A CLASS OF HIGH-ORDER IMEX SCHEMES FOR NAVIER-STOKES EQUATIONS WITH PERIODIC BOUNDARY CONDITIONS
}

\author{
FUKENG HUANG AND JIE SHEN
}

\begin{abstract}
We construct high-order semi-discrete-in-time and fully discrete (with Fourier-Galerkin in space) schemes for the incompressible Navier-Stokes equations with periodic boundary conditions, and carry out corresponding error analysis. The schemes are of implicit-explicit type based on a scalar auxiliary variable (SAV) approach. It is shown that numerical solutions of these schemes are uniformly bounded without any restriction on time step size. These uniform bounds enable us to carry out a rigorous error analysis for the schemes up to fifth-order in a unified form, and derive global error estimates in $l^{\infty}\left(0, T ; H^{1}\right) \cap l^{2}\left(0, T ; H^{2}\right)$ in the two dimensional case as well as local error estimates in $l^{\infty}\left(0, T ; H^{1}\right) \cap l^{2}\left(0, T ; H^{2}\right)$ in the three dimensional case. We also present numerical results confirming our theoretical convergence rates and demonstrating advantages of higher-order schemes for flows with complex structures in the double shear layer problem.
\end{abstract}

\section{INTRODUCTION}

Numerical approximation of the Navier-Stokes equations has been a subject of intensive study for many decades and continues to attract considerable attention, as it plays a fundamental role in computational fluid dynamics. Most of the work are concerned with the Navier-Stokes equations with non periodic boundary conditions, as is the case with the most applications. An enormous amount of work has been devoted to construct efficient and stable numerical algorithms for solving the incompressible Navier-Stokes equations with non periodic boundary conditions, see [12, 34, 7, 13, 16, 29] and the references therein. In particular, the papers 22, 20, 10, 15, 18, 6, among others, are particularly concerned with the error estimates for semi-discrete-in-time or fully discrete schemes.

We consider in this paper numerical approximation of the incompressible Navier-Stokes equations in primitive formulation:

$$
\begin{aligned}
& \frac{\partial \boldsymbol{u}}{\partial t}-\nu \Delta \boldsymbol{u}+(\boldsymbol{u} \cdot \nabla) \boldsymbol{u}+\nabla p=0, \\
& \nabla \cdot \boldsymbol{u}=0,
\end{aligned}
$$

with a suitable initial condition $\left.\boldsymbol{u}\right|_{t=0}=\boldsymbol{u}_{0}$ in a rectangular domain $\Omega \subset \mathbb{R}^{d}(d=2,3)$ with periodic boundary conditions. The unknowns are velocity $\boldsymbol{u}$ and the pressure $p$ which is assumed to have zero mean for uniqueness, $\nu>0$ is the viscosity. To simplify the presentation, we have set the external force to be zero. But our schemes and analytical results can be naturally extended to the case with a non-zero external force.

The incompressible Navier-Stokes equations with periodic boundary conditions retain the essential mathematical properties/difficulties of the system with non periodic boundary conditions, but are amenable to very efficient numerical algorithms using the Fourier-spectral method, and are particularly useful in the study of homogeneous turbulence [28, 30, 26].

There exists also a significant number of work devoted to the numerical analysis for Navier-Stokes equations with periodic boundary conditions. For examples, in [17, Hald proved the convergence

2000 Mathematics Subject Classification. 65M15; 76D05; 65M70.

Key words and phrases. Navier-stokes, stability, error analysis, high-order.

Department of Mathematics, Purdue University. This research is partially supported by NSF grants DMS-2012585 and AFOSR FA9550-20-1-0309. Emails: huang972@purdue.edu (F. Huang), shen7@purdue.edu (J. Shen). 
of semi-discrete Fourier-Galerkin methods in two and three dimensions; in [9, E used semigroup theory to establish convergence and error estimates of the semi-discrete Fourier-Galerkin and Fouriercollocation methods in various energy norms and $L^{p}$-norms; in [36], Wang proved uniform bounds and convergence of long time statistics for a semi-discrete second-order implicit-explicit (IMEX) scheme for the 2-D Navier-Stokes equations with periodic boundary conditions in vorticity-stream function formulation, see also related work in [14, 35]; in [5], Cheng and Wang established uniform bounds for semi-discrete higher-order (up to fourth-order) IMEX scheme for the 2-D Navier-Stokes equations with periodic boundary conditions in vorticity-stream function formulation; in [19, Heister et al. proved uniform bounds for a fully discrete finite-element and second-order IMEX scheme for the 2-D Navier-Stokes equations with periodic boundary conditions in vorticity-velocity formulation. Note that the uniform bounds for semi-discrete IMEX schemes obtained in the above references are for two-dimensional cases only and require that the time step be sufficiently small.

It appears that, except some recently constructed schemes based on the scalar auxiliary variable (SAV) approach 24, 23, all other IMEX type schemes (i.e., the nonlinear term is treated explicitly) for Navier-Stokes equations require the time step to be sufficiently small to have a bounded numerical solution. Furthermore, to the best of our knowledge, there is no error analysis for any IMEX scheme for the three-dimensional Navier-Stokes equations, and no error estimate is available for any higherorder $(\geq 3)$ IMEX scheme.

In this paper, we construct semi-discrete and fully discrete with Fourier-Galerkin in space SAV IMEX schemes and carry out a unified stability and error analysis. Our main contributions include:

- Our semi-discrete and fully discrete schemes of arbitrary order in time are unconditionally stable without any restriction on time step size;

- Global error estimates in $l^{\infty}\left(0, T ; H^{1}\right) \cap l^{2}\left(0, T ; H^{2}\right)$ up to fifth-order in time are established for the two-dimensional case;

- Local error estimates in $l^{\infty}\left(0, T_{*} ; H^{1}\right) \cap l^{2}\left(0, T_{*} ; H^{2}\right)$ (with a $\left.T_{*} \leq T\right)$ up to fifth-order in time are established for the three-dimensional case.

Our schemes are constructed using the SAV approach proposed in 21] which can be used for general dissipative systems. The main advantages of this approach, compared with other SAV approaches proposed in 24, 23. for Navier-Stokes equations is that our schemes are linear, decoupled and can be high-order. Moreover, in the two dimensional case, we use a stronger energy dissipation law (2.6), which is only true for the 2-D Navier-Stokes equations with periodic boundary conditions, that leads to a uniform bound for the numerical solution in $l^{\infty}\left(0, T ; H^{1}\right)$, as opposed to $l^{\infty}\left(0, T ; L^{2}\right)$ in the three dimensional case.

The rest of the paper is organized as follows. In the next section, we provide some preliminaries to be used in the sequel. In Section 3, we describe our semi-discrete and fully discrete with FourierGalerkin SAV schemes for the Navier-Stokes equations with periodic boundary condition, prove its unconditionally stability, and provide some numerical results to demonstrate the convergence rates and validate the robustness of our schemes. In section 4, we present detailed error analysis for the $k$ th-order schemes $(k=1,2,3,4,5)$ in a unified form. Some concluding remarks are given in the last section.

\section{Preliminaries}

We first introduce some notations. We denote by $(\cdot, \cdot)$ and $\|\cdot\|$ the inner product and the norm in $L^{2}(\Omega)$, and denote

$$
\boldsymbol{H}_{p}^{k}(\Omega)=\left\{u^{j}(j=0,1, \cdots, k) \in L^{2}(\Omega): u^{j}(j=0,1, \cdots, k-1) \quad \text { periodic, } \quad \int_{\Omega} u d \boldsymbol{x}=0\right\},
$$

with norm $\|\cdot\|_{k}$. For non-integer $s>0, H_{p}^{s}(\Omega)$ and the corresponding norm $\|\cdot\|_{s}$ are defined by space interpolation [1. In particular, we set $H_{p}^{0}(\Omega)=L_{0}^{2}(\Omega)$.

Let $V$ be a Banach space, we shall also use the standard notations $L^{p}(0, T ; V)$ and $C([0, T] ; V)$. To simplify the notation, we often omit the spatial dependence for the exact solution $u$, i.e., $u(x, t)$ 
is often denoted by $u(t)$. We shall use bold faced letters to denote vectors and vector spaces, and use $C$ to denote a generic positive constant independent of the discretization parameters.

We now define the following spaces which are particularly used for Navier-Stokes equations:

$$
\mathbf{H}=\left\{\boldsymbol{v} \in \boldsymbol{L}_{0}^{2}(\Omega): \nabla \cdot \boldsymbol{v}=0\right\}, \quad \mathbf{V}=\left\{\boldsymbol{v} \in \boldsymbol{H}_{p}^{1}(\Omega): \nabla \cdot \boldsymbol{v}=0\right\} .
$$

Let $\boldsymbol{v} \in \boldsymbol{L}_{0}^{2}(\Omega)$, we define $w:=\Delta^{-1} \boldsymbol{v}$ as the solution of

$$
\Delta \boldsymbol{w}=\boldsymbol{v} \quad \boldsymbol{x} \in \Omega ; \quad \boldsymbol{w} \text { periodic with zero mean. }
$$

Note that in the periodic case, we can define the operators $\nabla, \nabla \cdot$ and $\Delta^{-1}$ in the Fourier space by expanding functions and their derivatives in Fourier series, and one can easily show that these operators commute with each other.

We define a linear operator $\mathbf{A}$ in $\boldsymbol{L}_{0}^{2}(\Omega)$ by

$$
\mathbf{A} \boldsymbol{v}:=\nabla \times \nabla \times \Delta^{-1} \boldsymbol{v}, \quad \forall \boldsymbol{v} \in \boldsymbol{L}_{0}^{2}(\Omega) .
$$

Since

we derive immediately from the above that

$$
\|\Delta \boldsymbol{w}\|^{2}=\|\nabla \times \nabla \times \boldsymbol{w}\|^{2}+\|\nabla \nabla \cdot \boldsymbol{w}\|^{2} \quad \forall \boldsymbol{w} \in \boldsymbol{H}_{p}^{2}(\Omega),
$$

$$
\|\mathbf{A} \boldsymbol{v}\|=\left\|\Delta \Delta^{-1} \boldsymbol{v}\right\|-\left\|\nabla \nabla \cdot \Delta^{-1} \boldsymbol{v}\right\| \leq\|\boldsymbol{v}\|, \quad \forall \boldsymbol{v} \in \boldsymbol{L}_{0}^{2}(\Omega) .
$$

Next, we define the trilinear form $b(\cdot, \cdot, \cdot)$ and $b_{\mathbf{A}}(\cdot, \cdot, \cdot)$ by

$$
b(\boldsymbol{u}, \boldsymbol{v}, \boldsymbol{w})=\int_{\Omega}(\boldsymbol{u} \cdot \nabla) \boldsymbol{v} \cdot \boldsymbol{w} d \boldsymbol{x}, b_{\mathbf{A}}(\boldsymbol{u}, \boldsymbol{v}, \boldsymbol{w})=\int_{\Omega} \mathbf{A}((\boldsymbol{u} \cdot \nabla) \boldsymbol{v}) \cdot \boldsymbol{w} d \boldsymbol{x} .
$$

In particular, we have

$$
b(\boldsymbol{u}, \boldsymbol{v}, \boldsymbol{w})=-b(\boldsymbol{u}, \boldsymbol{w}, \boldsymbol{v}), \forall \boldsymbol{u} \in \mathbf{H}, \boldsymbol{v}, \boldsymbol{w} \in \boldsymbol{H}_{p}^{1}(\Omega),
$$

which implies

$$
b(\boldsymbol{u}, \boldsymbol{v}, \boldsymbol{v})=0, \forall \boldsymbol{u} \in \mathbf{H}, \boldsymbol{v} \in \boldsymbol{H}_{p}^{1}(\Omega) .
$$

In the two-dimensional periodic case, we have also 33

$$
b(\boldsymbol{u}, \boldsymbol{u}, \Delta \boldsymbol{u})=0, \forall \boldsymbol{u} \in \boldsymbol{H}_{p}^{2}(\Omega) .
$$

Taking the inner product of (1.1) with $\boldsymbol{u}$, thanks to 2.3), we find that solution of the Navier-Stokes equations (1.1) satisfies the energy dissipation law

$$
\frac{1}{2} \frac{d}{d t}\|\boldsymbol{u}\|^{2}=-\nu\|\nabla \boldsymbol{u}\|^{2} \quad(d=2,3) .
$$

On the other hand, in the two dimensional periodic case, taking the inner product of (1.1) with $-\Delta \boldsymbol{u}$, thanks to 2.4, we derive another energy dissipation law 33]

$$
\frac{1}{2} \frac{d}{d t}\|\nabla \boldsymbol{u}\|^{2}=-\nu\|\Delta \boldsymbol{u}\|^{2} \quad(d=2) .
$$

Using (2.2), Hölder inequality and Sobolev inequality, we have [33.

$$
\begin{aligned}
& b(\boldsymbol{u}, \boldsymbol{v}, \boldsymbol{w}), b_{\mathbf{A}}(\boldsymbol{u}, \boldsymbol{v}, \boldsymbol{w}) \leq c\|\mathbf{u}\|_{1}^{1 / 2}\|\mathbf{u}\|^{1 / 2}\|\mathbf{v}\|_{2}^{1 / 2}\|\mathbf{v}\|_{1}^{1 / 2}\|\mathbf{w}\|, \quad d=2 \\
& b(\boldsymbol{u}, \boldsymbol{v}, \boldsymbol{w}), b_{\mathbf{A}}(\boldsymbol{u}, \boldsymbol{v}, \boldsymbol{w}) \leq c\|\boldsymbol{u}\|_{1}\|\nabla \boldsymbol{v}\|_{1 / 2}\|\boldsymbol{w}\|, \quad d=3 .
\end{aligned}
$$

We also use frequently the following inequalities [33]:

$$
b(\boldsymbol{u}, \boldsymbol{v}, \boldsymbol{w}), b_{\mathbf{A}}(\boldsymbol{u}, \boldsymbol{v}, \boldsymbol{w}) \leq\left\{\begin{array}{l}
c\|\boldsymbol{u}\|_{1}\|\boldsymbol{v}\|_{1}\|\boldsymbol{w}\|_{1} ; \\
c\|\boldsymbol{u}\|_{2}\|\boldsymbol{v}\|_{0}\|\boldsymbol{w}\|_{1} ; \\
c\|\boldsymbol{u}\|_{2}\|\boldsymbol{v}\|_{1}\|\boldsymbol{w}\|_{0} ; \quad c \quad d \quad \boldsymbol{u}\left\|_{1}\right\| \boldsymbol{v}\left\|_{2}\right\| \boldsymbol{w} \|_{0} ; \\
c\|\boldsymbol{u}\|_{0}\|\boldsymbol{v}\|_{2}\|\boldsymbol{w}\|_{1} ;
\end{array} \quad d \leq 4 .\right.
$$

Note that (2.4), (2.6), and (2.7) enable us to obtain global error estimates in the two-dimensional case. 


\section{The SAV SCHEMES AND STABILITy RESUlts}

In this section, we construct semi-discrete and fully discrete SAV schemes for the incompressible Navier-Stokes equations, and establish stability results for both semi-discrete and fully discrete schemes. More precisely, we shall prove uniform $L^{2}$ bound for the SAV scheme based on the dissipation law 2.5 in $3 \mathrm{D}$ case, and prove a uniform $H^{1}$ bound for the SAV scheme based on the dissipation law 2.6 in $2 \mathrm{D}$ case.

3.1. The SAV schemes. Following the ideas in [21 for the general dissipative systems, we construct below unconditionally energy stable schemes for (1.1).

For Navier-Stokes equations with periodic boundary conditions, we can explicitly eliminate the pressure from (1.1). Indeed, taking the divergence on both sides of (1.1), we find

$$
-\Delta p=\nabla \cdot(\boldsymbol{u} \cdot \nabla \boldsymbol{u})
$$

from which we derive

$$
\begin{aligned}
\nabla p & =\nabla \Delta^{-1} \Delta p=-\nabla \Delta^{-1} \nabla \cdot(\boldsymbol{u} \cdot \nabla \boldsymbol{u}) \\
& =-\nabla \nabla \cdot \Delta^{-1}(\boldsymbol{u} \cdot \nabla \boldsymbol{u})=-(\Delta+\nabla \times \nabla \times) \Delta^{-1}(\boldsymbol{u} \cdot \nabla \boldsymbol{u}) \\
& =-\boldsymbol{u} \cdot \nabla \boldsymbol{u}-\nabla \times \nabla \times \Delta^{-1}(\boldsymbol{u} \cdot \nabla \boldsymbol{u})=-\boldsymbol{u} \cdot \nabla \boldsymbol{u}-\mathbf{A}(\boldsymbol{u} \cdot \nabla \boldsymbol{u}),
\end{aligned}
$$

where $\mathbf{A}$ is defined in 2.1. Hence, 1.1 is equivalent to 3.1 and

$$
\frac{\partial \boldsymbol{u}}{\partial t}-\nu \Delta \boldsymbol{u}-\mathbf{A}(\boldsymbol{u} \cdot \nabla \boldsymbol{u})=0
$$

In order to apply the SAV approach, we introduce a SAV, $r(t)=E(\boldsymbol{u}(t))+1$, and expand 3.3 as

$$
\begin{aligned}
& \frac{\partial \boldsymbol{u}}{\partial t}-\nu \Delta \boldsymbol{u}-\mathbf{A}(\boldsymbol{u} \cdot \nabla \boldsymbol{u})=0, \\
& \frac{d E}{d t}=\left\{\begin{array}{cc}
-\nu \frac{r(t)}{E(\boldsymbol{u}(t))+1}\|\Delta \boldsymbol{u}\|^{2}, & d=2, \\
-\nu \frac{r(t)}{E(\boldsymbol{u}(t))+1}\|\nabla \boldsymbol{u}\|^{2}, & d=3,
\end{array}\right.
\end{aligned}
$$

where

$$
E(\boldsymbol{u})= \begin{cases}\frac{1}{2}\|\nabla \boldsymbol{u}\|^{2}, & d=2, \\ \frac{1}{2}\|\boldsymbol{u}\|^{2}, & d=3 .\end{cases}
$$

We construct below semi-discrete and fully discrete schemes for the expanded system (3.4).

3.1.1. Semi-discrete $S A V$ schemes. We consider first the time discretization of (3.4) based on the implicit-explicit BDF- $k$ formulae in the following unified form:

Given $r^{n}, \boldsymbol{u}^{j}(j=n, n-1, \cdots, n-k+1)$, we compute $\overline{\boldsymbol{u}}^{n+1}, r^{n+1}, p^{n+1}, \xi^{n+1}$ and $\boldsymbol{u}^{n+1}$ consecutively by

$$
\begin{aligned}
& \frac{\alpha_{k} \overline{\boldsymbol{u}}^{n+1}-A_{k}\left(\overline{\boldsymbol{u}}^{n}\right)}{\delta t}-\nu \Delta \overline{\boldsymbol{u}}^{n+1}-\mathbf{A}\left(B_{k}\left(\boldsymbol{u}^{n}\right) \cdot \nabla B_{k}\left(\boldsymbol{u}^{n}\right)\right)=0, \\
& \frac{1}{\delta t}\left(r^{n+1}-r^{n}\right)=\left\{\begin{array}{cc}
-\nu \frac{r^{n+1}}{E\left(\overline{\boldsymbol{u}}^{n+1}\right)+1}\left\|\Delta \overline{\boldsymbol{u}}^{n+1}\right\|^{2}, & d=2, \\
-\nu \frac{r^{n+1}}{E\left(\overline{\boldsymbol{u}}^{n+1}\right)+1}\left\|\nabla \overline{\boldsymbol{u}}^{n+1}\right\|^{2}, & d=3 ;
\end{array}\right. \\
& \xi^{n+1}=\frac{r^{n+1}}{E\left(\overline{\boldsymbol{u}}^{n+1}\right)} ; \\
& \boldsymbol{u}^{n+1}=\eta_{k}^{n+1} \overline{\boldsymbol{u}}^{n+1} \text { with } \eta_{k}^{n+1}=1-\left(1-\xi^{n+1}\right)^{k} .
\end{aligned}
$$

Whenever pressure is needed, it can be computed from

$$
\Delta p^{n+1}=-\nabla \cdot\left(\boldsymbol{u}^{n+1} \cdot \nabla \boldsymbol{u}^{n+1}\right)
$$

In the above, $\alpha_{k}$, the operators $A_{k}$ and $B_{k}(k=1,2,3,4,5)$ are given by: 
first-order:

$$
\alpha_{1}=1, \quad A_{1}\left(\boldsymbol{u}^{n}\right)=\boldsymbol{u}^{n}, \quad B_{1}\left(\overline{\boldsymbol{u}}^{n}\right)=\bar{u}^{n}
$$

second-order:

$$
\alpha_{2}=\frac{3}{2}, \quad A_{2}\left(\boldsymbol{u}^{n}\right)=2 \boldsymbol{u}^{n}-\frac{1}{2} \boldsymbol{u}^{n-1}, \quad B_{2}\left(\overline{\boldsymbol{u}}^{n}\right)=2 \overline{\boldsymbol{u}}^{n}-\overline{\boldsymbol{u}}^{n-1}
$$

third-order:

$$
\alpha_{3}=\frac{11}{6}, \quad A_{3}\left(\boldsymbol{u}^{n}\right)=3 \boldsymbol{u}^{n}-\frac{3}{2} \boldsymbol{u}^{n-1}+\frac{1}{3} \boldsymbol{u}^{n-2}, \quad B_{3}\left(\overline{\boldsymbol{u}}^{n}\right)=3 \overline{\boldsymbol{u}}^{n}-3 \overline{\boldsymbol{u}}^{n-1}+\overline{\boldsymbol{u}}^{n-2}
$$

fourth-order:

$$
\alpha_{4}=\frac{25}{12}, A_{4}\left(\boldsymbol{u}^{n}\right)=4 \boldsymbol{u}^{n}-3 \boldsymbol{u}^{n-1}+\frac{4}{3} \boldsymbol{u}^{n-2}-\frac{1}{4} \boldsymbol{u}^{n-3}, B_{4}\left(\overline{\boldsymbol{u}}^{n}\right)=4 \overline{\boldsymbol{u}}^{n}-6 \overline{\boldsymbol{u}}^{n-1}+4 \overline{\boldsymbol{u}}^{n-2}-\overline{\boldsymbol{u}}^{n-3} ;
$$

fifth-order:

$$
\begin{aligned}
\alpha_{5}=\frac{137}{60}, \quad A_{5}\left(\boldsymbol{u}^{n}\right) & =5 \boldsymbol{u}^{n}-5 \boldsymbol{u}^{n-1}+\frac{10}{3} \boldsymbol{u}^{n-2}-\frac{5}{4} \boldsymbol{u}^{n-3}+\frac{1}{5} \boldsymbol{u}^{n-4} \\
B_{5}\left(\overline{\boldsymbol{u}}^{n}\right) & =5 \overline{\boldsymbol{u}}^{n}-10 \overline{\boldsymbol{u}}^{n-1}+10 \overline{\boldsymbol{u}}^{n-2}-5 \overline{\boldsymbol{u}}^{n-3}+\overline{\boldsymbol{u}}^{n-4}
\end{aligned}
$$

Several remarks are in order:

- We observe from $3.6 \mathrm{~b}$ that $r^{n+1}$ is a first-order approximation to $E\left(u\left(\cdot, t_{n+1}\right)\right)+1$ which implies that $\xi^{n+1}$ is a first-order approximation to 1.

- $3.6 \mathrm{a}$ ) is a $k$ th-order approximation to 3.3 with $k$ th-order BDF for the linear terms and $k$ thorder Adams-Bashforth extrapolation for the nonlinear terms. Hence, $\overline{\boldsymbol{u}}^{n+1}$ is a $k$ th-order approximation to $\boldsymbol{u}\left(\cdot, t^{n+1}\right)$, which, along with $3.6 \mathrm{~b}$ ) and $\left.3.6 \mathrm{a}\right)$, implies that $\boldsymbol{u}^{n+1}$ and $p^{n+1}$ are $k$ th-order approximations for $\boldsymbol{u}\left(\cdot, t^{n+1}\right)$ and $p\left(\cdot, t^{n+1}\right)$.

- The main computational cost is to solve the Poisson type equation 3.6a).

3.1.2. Fully discrete schemes with Fourier spectral method in space. We now consider $\Omega=\left[0, L_{x}\right) \times$ $\left[0, L_{y}\right) \times\left[0, L_{z}\right)$ with periodic boundary conditions. We partition the domain $\Omega=\left(0, L_{x}\right) \times\left(0, L_{y}\right) \times$ $\left(0, L_{z}\right)$ uniformly with size $h_{x}=L_{x} / N_{x}, h_{y}=L_{y} / N_{y}, h_{z}=L_{z} / N_{z}$ and $N x, N y, N z$ are positive even integers. Then the Fourier approximation space can be defined as

$$
S_{N}=\operatorname{span}\left\{e^{i \xi_{j} x} e^{i \eta_{k} y} e^{i \tau_{l} z}:-\frac{N_{x}}{2} \leq j \leq \frac{N_{x}}{2}-1,-\frac{N_{y}}{2} \leq k \leq \frac{N_{y}}{2}-1,-\frac{N_{z}}{2} \leq l \leq \frac{N_{z}}{2}-1\right\} \backslash \mathbb{R},
$$

where $i=\sqrt{-1}, \xi_{j}=2 \pi j / L_{x}, \eta_{k}=2 \pi k / L_{y}$ and $\tau_{l}=2 \pi l / L_{z}$. Then, any function $u(x, y, z) \in L^{2}(\Omega)$ can be approximated by:

$$
u(x, y, z) \approx u_{N}(x, y, z)=\sum_{j=-\frac{N_{x}}{2}}^{\frac{N x}{2}-1} \sum_{k=-\frac{N_{y}}{2}}^{\frac{N_{y}}{2}-1} \sum_{l=-\frac{N_{z}}{2}}^{\frac{N_{z}}{2}-1} \hat{u}_{j, k, l} e^{i \xi_{j} x} e^{i \eta_{k} y} e^{i \tau_{l} z},
$$

with the Fourier coefficients defined as

$$
\hat{u}_{j, k, l}=\frac{1}{|\Omega|} \int_{\Omega} u e^{-i\left(\xi_{j} x+\eta_{k} y+\tau_{l} z\right)} d \boldsymbol{x} .
$$

In the following, we fix $N_{x}=N_{y}=N_{z}=N$ for simplicity.

Define the $L^{2}$-orthogonal projection operator $\Pi_{N}: L^{2}(\Omega) \rightarrow S_{N}$ by

$$
\left(\Pi_{N} u-u, \Psi\right)=0, \quad \forall \Psi \in S_{N}, \quad u \in L^{2}(\Omega),
$$

then we have the following approximation results (cf. [22]):

Lemma 1. For any $0 \leq k \leq m$, there exists a constant $C$ such that

$$
\left\|\Pi_{N} u-u\right\|_{k} \leq C\|u\|_{m} N^{k-m}, \forall u \in \boldsymbol{H}_{p}^{m}(\Omega) .
$$


We are now ready to describe our fully discrete schemes.

Given $r^{n}$ and $\boldsymbol{u}_{N}^{j} \in S_{N}$ for $j=n, \ldots, n-k+1$, we compute $\overline{\boldsymbol{u}}_{N}^{n+1}, r^{n+1}, p_{N}^{n+1}, \xi^{n+1}$ and $\boldsymbol{u}_{N}^{n+1}$ consecutively by

$$
\begin{aligned}
& \left(\frac{\alpha_{k} \overline{\boldsymbol{u}}_{N}^{n+1}-A_{k}\left(\overline{\boldsymbol{u}}_{N}^{n}\right)}{\delta t}, v_{N}\right)+\nu\left(\nabla \overline{\boldsymbol{u}}_{N}^{n+1}, \nabla v_{N}\right)-\left(\mathbf{A}\left(B_{k}\left(\boldsymbol{u}_{N}^{n}\right) \cdot \nabla B_{k}\left(\boldsymbol{u}_{N}^{n}\right)\right), v_{N}\right)=0, \quad \forall v_{N} \in S_{N} \\
& \frac{1}{\delta t}\left(r^{n+1}-r^{n}\right)= \begin{cases}-\nu \frac{r^{n+1}}{E\left(\overline{\boldsymbol{u}}_{N}^{n+1}\right)+1}\left\|\Delta \overline{\boldsymbol{u}}_{N}^{n+1}\right\|^{2}, & d=2, \\
-\nu \frac{r^{n+1}}{E\left(\overline{\boldsymbol{u}}_{N}^{n+1}\right)+1}\left\|\nabla \overline{\boldsymbol{u}}_{N}^{n+1}\right\|^{2}, & d=3 ;\end{cases} \\
& \xi^{n+1}=\frac{r^{n+1}}{E\left(\overline{\boldsymbol{u}}_{N}^{n+1}\right)+1} ; \\
& \boldsymbol{u}_{N}^{n+1}=\eta_{k}^{n+1} \overline{\boldsymbol{u}}_{N}^{n+1} \text { with } \eta_{k}^{n+1}=1-\left(1-\xi^{n+1}\right)^{k},
\end{aligned}
$$

where $\alpha_{k}$, the operators $A_{k}$ and $B_{k}(k=1,2,3,4,5)$ are given in $(3.8)$ - $(3.12)$.

Note that Fourier approximation of Poisson type equations leads to diagonal matrix in the frequency space, so the above scheme can be efficiently implemented as follows:

(i) Compute $\overline{\boldsymbol{u}}_{N}^{n+1}$ from (3.14a), which is a Poisson-type equation;

(ii) With $\overline{\boldsymbol{u}}_{N}^{n+1}$ known, determine $r^{n+1}$ explicitly from (3.14b);

(iii) Compute $\xi^{n+1}, \eta_{k}^{n+1}$ and $\boldsymbol{u}_{N}^{n+1}$ from (3.14c) and (3.14d), goto the next step.

Finally, whenever pressure is needed, it can be computed from

$$
\Delta p_{N}^{n+1}=-\Pi_{N} \nabla \cdot\left(\boldsymbol{u}_{N}^{n+1} \cdot \nabla \boldsymbol{u}_{N}^{n+1}\right) .
$$

3.2. Stability results. We have the following results concerning the stability of the above schemes.

Theorem 1. Let $\boldsymbol{u}_{0} \in \boldsymbol{V} \cap \boldsymbol{H}_{p}^{2}$ if $d=2$ and $\boldsymbol{u}_{0} \in \boldsymbol{V}$ if $d=3$. Let $\left\{r^{k}, \xi^{k}, \overline{\boldsymbol{u}}_{N}^{k}, \boldsymbol{u}_{N}^{k}\right\}$ be the solution of the fully discrete scheme (3.14). Then, given $r^{n} \geq 0$, we have $r^{n+1} \geq 0, \xi^{n+1} \geq 0$, and for any $k$, the scheme (3.14) is unconditionally energy stable in the sense that

$$
r^{n+1}-r^{n}=\left\{\begin{array}{ll}
-\delta t \nu \xi^{n+1}\left\|\Delta \overline{\boldsymbol{u}}_{N}^{n+1}\right\|^{2} \leq 0, & d=2, \\
-\delta t \nu \xi^{n+1}\left\|\nabla \overline{\boldsymbol{u}}_{N}^{n+1}\right\|^{2} \leq 0, & d=3,
\end{array} \quad \forall n .\right.
$$

Furthermore, there exists $M_{k}>0$ such that

$$
\begin{aligned}
& \left\|\nabla \boldsymbol{u}_{N}^{n+1}\right\|^{2} \leq M_{k}^{2}, \quad d=2, \quad \forall n \\
& \left\|\boldsymbol{u}_{N}^{n+1}\right\|^{2} \leq M_{k}^{2}, \quad d=3,
\end{aligned}
$$

Same results hold for the semi-discrete schemes (3.6) with $\overline{\boldsymbol{u}}_{N}^{n+1}$ and $\boldsymbol{u}_{N}^{n+1}$ in (3.16) and (3.17) be replaced by $\overline{\boldsymbol{u}}^{n+1}$ and $\boldsymbol{u}^{n+1}$.

Proof. Since the proofs for the fully discrete scheme (3.14) and for the semi-discrete scheme (3.6) are essentially the same, we shall only give the proof for the fully discrete scheme (3.14) below.

Given $r^{n} \geq 0$. Since $E\left(\overline{\boldsymbol{u}}_{N}^{n+1}\right)>0$, it follows from $3.14 \mathrm{~b}$ that

$$
r^{n+1}= \begin{cases}\frac{r^{n}}{1+\delta t \nu \frac{\left\|\Delta \bar{u}_{N}^{n+1}\right\|^{2}}{E\left(\bar{u}_{N}^{n+1}\right)+1}} \geq 0, & d=2, \\ \frac{r^{n}}{1+\delta t \nu \frac{\left\|\nabla \bar{u}_{N}^{n+1}\right\|^{2}}{E\left(\bar{u}_{N}^{N+1}\right)+1}} \geq 0, & d=3 .\end{cases}
$$

Then we derive from $3.14 \mathrm{c}$ ) that $\xi^{n+1} \geq 0$ and obtain (3.16).

Denote $M:=r^{0}=E[\boldsymbol{u}(\cdot, 0)]$, then (3.16) implies $r^{n} \leq M, \forall n$. It then follows from 3.14c that

$$
\left|\xi^{n+1}\right|=\frac{r^{n+1}}{E\left(\overline{\boldsymbol{u}}_{N}^{n+1}\right)+1} \leq \begin{cases}\frac{2 M}{\left\|\nabla \overline{\boldsymbol{u}}_{N}^{n+1}\right\|^{2}+2}, & d=2, \\ \frac{2 M}{\left\|\overline{\boldsymbol{u}}_{N}^{n+1}\right\|^{2}+2}, & d=3 .\end{cases}
$$


Since $\eta_{k}^{n+1}=1-\left(1-\xi^{n+1}\right)^{k}$, we have $\eta_{k}^{n+1}=\xi^{n+1} P_{k-1}\left(\xi^{n+1}\right)$ with $P_{k-1}$ being a polynomial of degree $k-1$. Then, we derive from (3.18) that there exists $M_{k}>0$ such that

$$
\left|\eta_{k}^{n+1}\right|=\left|\xi^{n+1} P_{k-1}\left(\xi^{n+1}\right)\right| \leq\left\{\begin{array}{l}
\frac{M_{k}}{\left\|\nabla \overline{\boldsymbol{u}}_{N}^{n+1}\right\|^{2}+2}, \quad d=2, \\
\frac{M_{k}}{\left\|\overline{\boldsymbol{u}}_{N}^{n+1}\right\|^{2}+2}, \quad d=3,
\end{array}\right.
$$

which, along with $\boldsymbol{u}_{N}^{n+1}=\eta_{k}^{n+1} \overline{\boldsymbol{u}}_{N}^{n+1}$, implies

$$
\begin{aligned}
& \left\|\nabla \boldsymbol{u}_{N}^{n+1}\right\|^{2}=\left(\eta_{k}^{n+1}\right)^{2}\left\|\nabla \overline{\boldsymbol{u}}_{N}^{n+1}\right\|^{2} \leq\left(\frac{M_{k}}{\left\|\nabla \overline{\boldsymbol{u}}_{N}^{n+1}\right\|^{2}+2}\right)^{2}\left\|\nabla \overline{\boldsymbol{u}}_{N}^{n+1}\right\|^{2} \leq M_{k}^{2}, \quad d=2, \\
& \left\|\boldsymbol{u}_{N}^{n+1}\right\|^{2}=\left(\eta_{k}^{n+1}\right)^{2}\left\|\overline{\boldsymbol{u}}_{N}^{n+1}\right\|^{2} \leq\left(\frac{M_{k}}{\left\|\overline{\boldsymbol{u}}_{N}^{n+1}\right\|^{2}+2}\right)^{2}\left\|\overline{\boldsymbol{u}}_{N}^{n+1}\right\|^{2} \leq M_{k}^{2}, \quad d=3 .
\end{aligned}
$$

3.3. Numerical examples. Before we start the error analysis, we provide numerical examples to demonstrate the convergence rates and compare the performance of the schemes with different orders on a classical benchmark problem.

Example 1: Convergence test. Consider the Navier-Stokes equations 1.1) with an external forcing $\boldsymbol{f}$ in $\Omega=(0,2) \times(0,2)$ with periodic boundary condition such that the exact solution is given by

$$
\begin{aligned}
& u_{1}(x, y)=\pi \exp (\sin (\pi x)) \exp (\sin (\pi y)) \cos (\pi y) \sin ^{2}(t) \\
& u_{2}(x, y)=-\pi \exp (\sin (\pi x)) \exp (\sin (\pi y)) \cos (\pi x) \sin ^{2}(t) \\
& p(x, y)=\exp (\cos (\pi x) \sin (\pi y)) \sin ^{2}(t) .
\end{aligned}
$$

We set $\nu=1$ in 1 1.1), and use the Fourier spectral method with $40 \times 40$ modes for space discretization so that the spatial discretization error is negligible with respect to the time discretization error. In Figures 1, we plot the convergence rate of the $H^{1}$ error for the velocity and the pressure at $T=1$ by using first- to fourth-order schemes. We observe the expected convergence rates for both the velocity and the pressure.

Example 2: Double shear layer problem [3, 4, 8]. Consider the Navier-Stokes equations (1.1) in $\Omega=(0,1) \times(0,1)$ with periodic boundary conditions and the initial condition given by

$$
\begin{aligned}
& u_{1}(x, y, 0)=\left\{\begin{array}{l}
\tanh (\rho(y-0.25)), y \leq 0.5 \\
\tanh (\rho(0.75-y)), y>0.5
\end{array}\right. \\
& u_{2}(x, y, 0)=\delta \sin (2 \pi x)
\end{aligned}
$$

where $\rho$ determines the slope of the shear layer and $\delta$ represents the size of the perturbation. In our simulations, we fix $\delta=0.05$. We first test a thick layer problem by choosing $\rho=30$ and $\nu=0.0001$. We use the Fourier spectral method with $128 \times 128$ modes for the space discretization, and set $\delta t=8 \times 10^{-4}$. In Figures 2, we show the vorticity contours at $T=1.2$ obtained with first- to fourth-order schemes. We observe that correct solution is obtained with the third- and fourth-order schemes while the first-order scheme gives totally wrong result and the second-order scheme leads to inaccurate result.

Next, we test a thin layer problem by choosing $\rho=100$ and $\nu=0.00005$. We use first- to the fourth-order schemes with $256 \times 256$ Fourier modes and $\delta t=3 \times 10^{-4}$. In Figures 3 , we plot the vorticity contours at $T=1.2$. We observe that correct solutions are obtained with the third- and fourth-order schemes while first- and second-order schemes lead to wrong results.

In order to examine the effect of SAV approach, we plot in Figure 4 evolution of the SAV factor $\eta=1-(1-\xi)^{2}$ and the vorticity contours at $T=1.2$, computed with the second-order scheme with $\delta t=2.5 \times 10^{-4}$. We observe that at around $t=1.05$, where the usual semi-implicit secondorder scheme blows up, the SAV factor dips slightly to allow the scheme continue to produce correct simulation. 


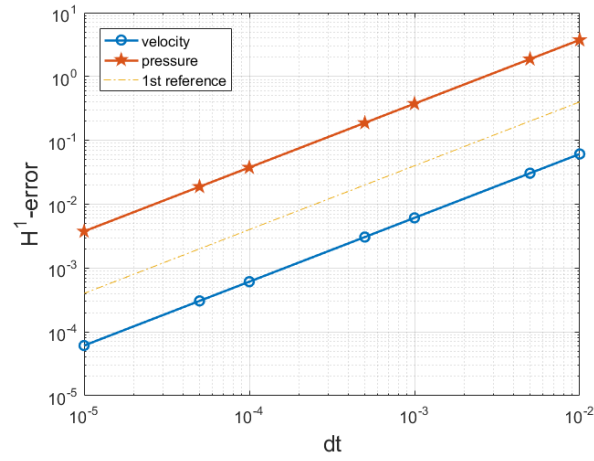

(a) BDF1 errors of velocity and pressure

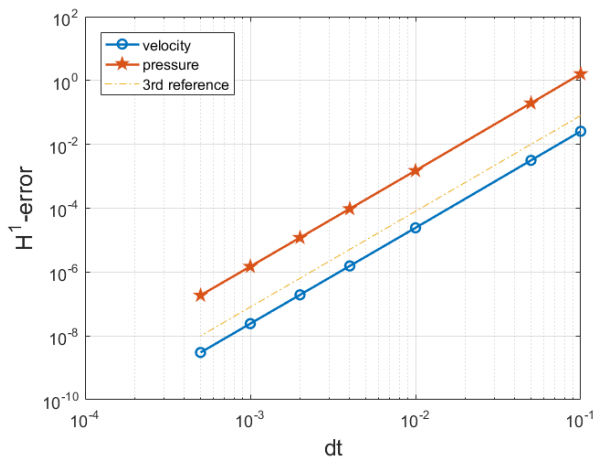

(c) BDF3 errors of velocity and pressure

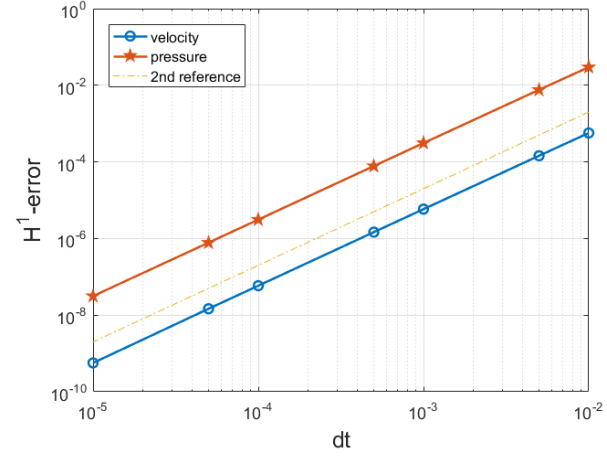

(b) BDF2 errors of velocity and pressure

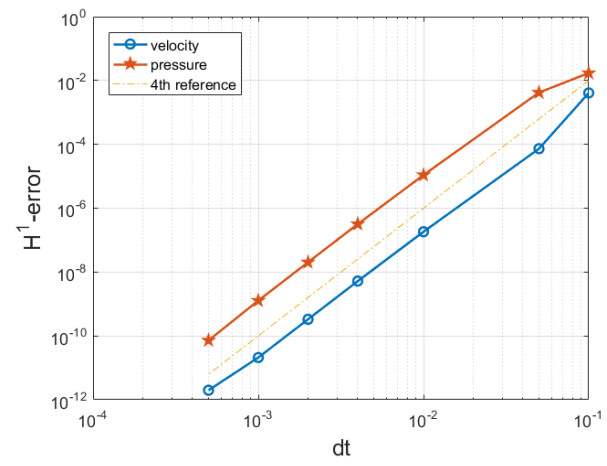

(d) BDF4 errors of velocity and pressure

FiguRE 1. Convergence test for the Navier-stokes equations using $\operatorname{SAV} / \operatorname{BDF} k(k=1,2,3,4)$

Remark 1. These two tests indicate that for high Reynolds number flows with complex structures, higher-order schemes are preferred over lower-order schemes, as much smaller time steps have to be used to obtain correct solutions with lower-order schemes.

Note that if we use the usual semi-implicit schemes with the same time steps in the above tests, the first- and second-order schemes would blow up. So the SAV approach can effectively prevent the numerical solution from blowing up although sufficient small time steps are needed to capture the correct solution. Thus, one is advised to adopt a suitable adaptive time stepping to take full advantage of the SAV schemes.

\section{ERror ANALYSIS}

In this section, we carry out a unified error analysis for the fully discrete schemes (3.14) with $1 \leq k \leq 5$, and state, as corollaries, similar results for the semi-discrete schemes (3.6).

We denote

$$
\begin{aligned}
& t^{n}=n \delta t, \quad s^{n}=r^{n}-r\left(t^{n}\right), \\
& \overline{\boldsymbol{e}}_{N}^{n}=\overline{\boldsymbol{u}}_{N}^{n}-\Pi_{N} \boldsymbol{u}\left(\cdot, t^{n}\right), \quad \boldsymbol{e}_{N}^{n}=\boldsymbol{u}_{N}^{n}-\Pi_{N} \boldsymbol{u}\left(\cdot, t^{n}\right), \quad \boldsymbol{e}_{\Pi}^{n}=\Pi_{N} \boldsymbol{u}\left(\cdot, t^{n}\right)-\boldsymbol{u}\left(\cdot, t^{n}\right), \\
& \overline{\boldsymbol{e}}^{n}=\overline{\boldsymbol{u}}_{N}^{n}-\boldsymbol{u}\left(\cdot, t^{n}\right)=\overline{\boldsymbol{e}}_{N}^{n}+\boldsymbol{e}_{\Pi}^{n}, \quad \boldsymbol{e}^{n}=\boldsymbol{u}_{N}^{n}-\boldsymbol{u}\left(\cdot, t^{n}\right)=\boldsymbol{e}_{N}^{n}+\boldsymbol{e}_{\Pi}^{n} .
\end{aligned}
$$

To simplify the notations, we dropped the dependence on $N$ for $\overline{\boldsymbol{e}}^{n}$ and $\boldsymbol{e}^{n}$ in the above, and will do so for some other quantities in the sequel. 


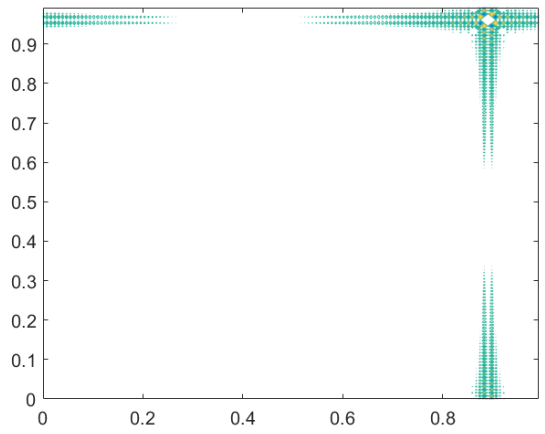

(a) 1st order

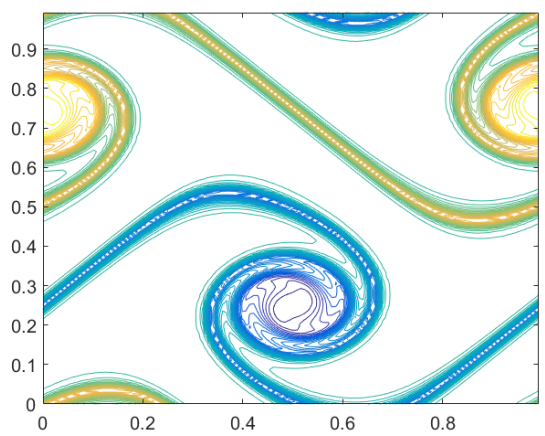

(c) 3rd-order

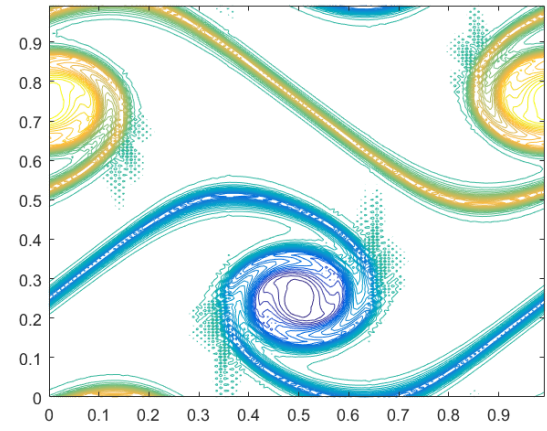

(b) 2nd-order

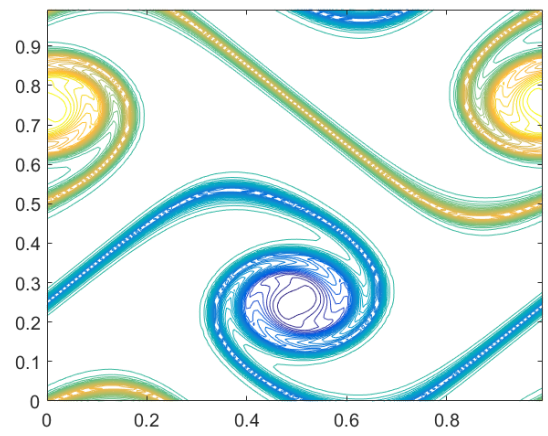

(d) 4th-order

FiguRE 2. Thick layer problem: vorticity contours at $\mathrm{T}=1.2$ with $\rho=30, \nu=0.0001$ and $\delta t=8 \times 10^{-4}$

4.1. Several useful lemmas. We will frequently use the following two discrete versions of the Gronwall lemma.

Lemma 2. (Discrete Gronwall Lemma 1 [32]) Let $y^{k}, h^{k}, g^{k}, f^{k}$ be four nonnegative sequences satisfying

$$
y^{n}+\delta t \sum_{k=0}^{n} h^{k} \leq B+\delta t \sum_{k=0}^{n}\left(g^{k} y^{k}+f^{k}\right) \quad \text { with } \delta t \sum_{k=0}^{T / \delta t} g^{k} \leq M, \forall 0 \leq n \leq T / \delta t .
$$

We assume $\delta t g^{k}<1$ for all $k$, and let $\sigma=\max _{0 \leq k \leq T / \delta t}\left(1-\delta t g^{k}\right)^{-1}$. Then

$$
y^{n}+\delta t \sum_{k=1}^{n} h^{k} \leq \exp (\sigma M)\left(B+\delta t \sum_{k=0}^{n} f^{k}\right), \forall n \leq T / \delta t .
$$

Lemma 3. (Discrete Gronwall Lemma 2 [31]) Let, $a_{n}, b_{n}, c_{n}$, and $d_{n}$ be four nonnegative sequences satisfying

$$
a_{m}+\tau \sum_{n=1}^{m} b_{n} \leq \tau \sum_{n=0}^{m-1} a_{n} d_{n}+\tau \sum_{n=0}^{m-1} c_{n}+C, m \geq 1
$$




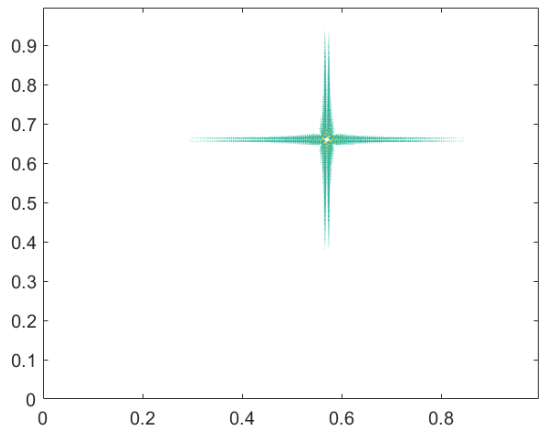

(a) 1st-order

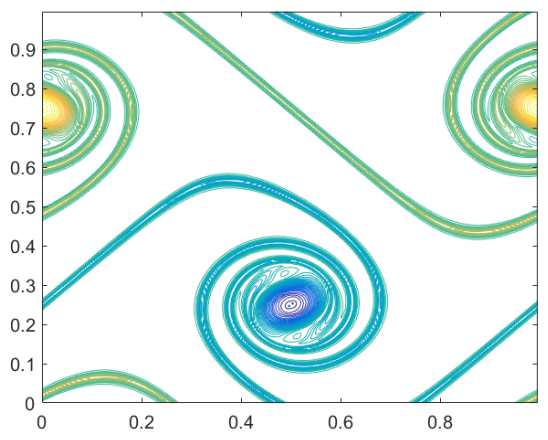

(c) 3rd-order

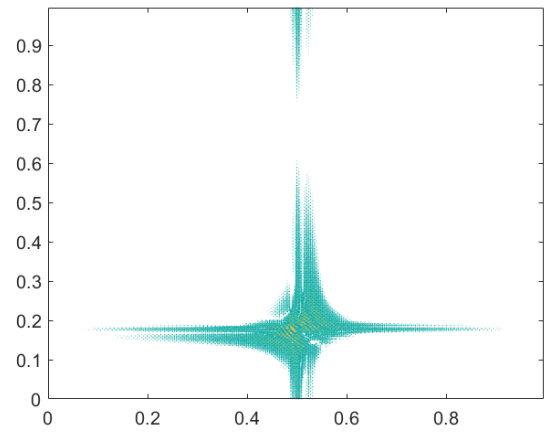

(b) 2nd-order

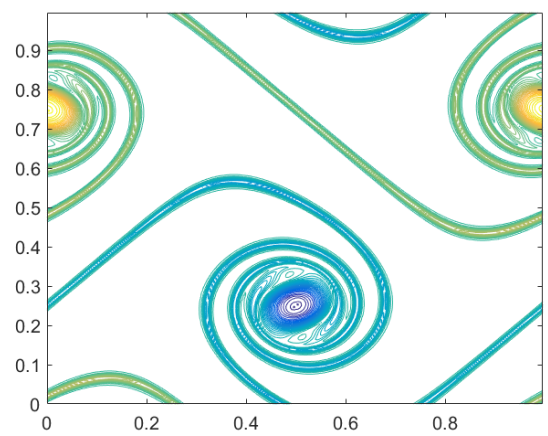

(d) 4th-order

FIGURE 3. Thin layer problem: vorticity contours at $\mathrm{T}=1.2$ with $\rho=100, \nu=0.00005$ and $\delta t=3 \times 10^{-4}$

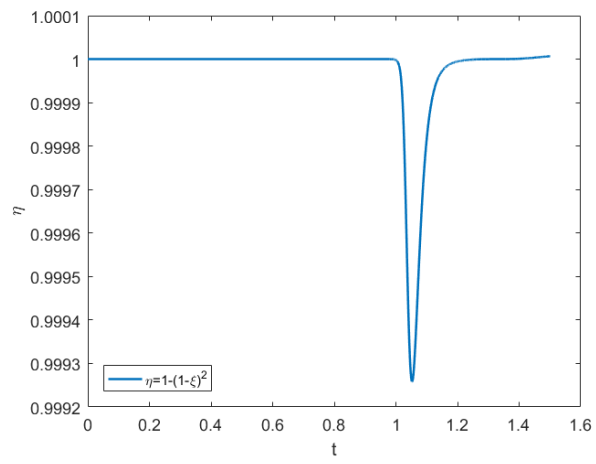

(a) Evolution of $\eta$

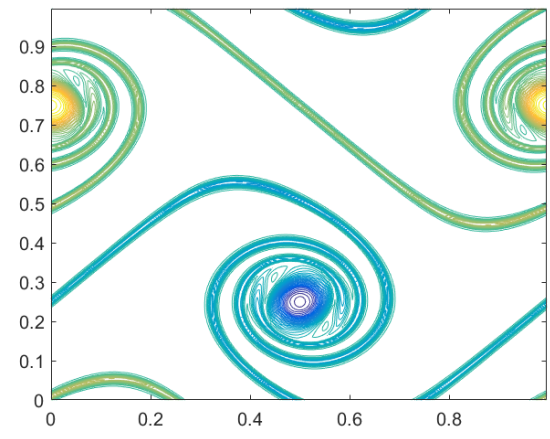

(b) Vorticity contours at $t=1.2$

FIGURE 4. Thin layer problem: second-order scheme with $\rho=100, \nu=0.00005$ and $\delta t=2.5 \times 10^{-4}$ 
where $C$ and $\tau$ are two positive constants. Then

$$
a_{m}+\tau \sum_{n=1}^{m} b_{n} \leq \exp \left(\tau \sum_{n=0}^{m-1} d_{n}\right)\left(\tau \sum_{n=0}^{m-1} c_{n}+C\right), m \geq 1
$$

Based on Dahlquist's G-stability theory, Nevanlinna and Odeh [27] proved the following result which plays an essential role in our error analysis.

Lemma 4. For $1 \leq k \leq 5$, there exist $0 \leq \tau_{k}<1$, a positive definite symmetric matrix $G=\left(g_{i j}\right) \in$ $\mathcal{R}^{k, k}$ and real numbers $\delta_{0}, \ldots, \delta_{k}$ such that

$$
\begin{aligned}
\left(\alpha_{k} u^{n+1}-A_{k}\left(u^{n}\right), u^{n+1}-\tau_{k} u^{n}\right) & =\sum_{i, j=1}^{k} g_{i j}\left(u^{n+1+i-k}, u^{n+1+j-k}\right) \\
& -\sum_{i, j=1}^{k} g_{i j}\left(u^{n+i-k}, u^{n+j-k}\right)+\left\|\sum_{i=0}^{k} \delta_{i} u^{n+1+i-k}\right\|^{2},
\end{aligned}
$$

where the smallest possible values of $\tau_{k}$ are

$$
\tau_{1}=\tau_{2}=0, \quad \tau_{3}=0.0836, \quad \tau_{4}=0.2878, \quad \tau_{5}=0.8160,
$$

and $\alpha_{k}, A_{k}$ are defined in $3.10-(3.12$.

We also recall the following lemma 25] which will be used to prove local error estimates in the three-dimensional case.

Lemma 5. Let $\phi:(0, \infty) \rightarrow(0, \infty)$ be continuous and increasing, and let $M>0$. Given $T_{*}$ such that $0<T_{*}<\int_{M}^{\infty} d z / \phi(z)$, there exists $C_{*}>0$ independent of $\delta t>0$ with the following property. Suppose that quantities $z_{n}, w_{n} \geq 0$ satisfy

$$
z_{n}+\sum_{k=0}^{n-1} \delta t w_{k} \leq y_{n}:=M+\sum_{k=0}^{n-1} \delta t \phi\left(z_{k}\right), \forall n \leq n_{*} .
$$

with $n_{*} \delta t \leq T_{*}$. Then $y_{n_{*}} \leq C_{*}$.

\subsection{Error analysis for the velocity in $2 \mathrm{D}$.}

Theorem 2. Let $d=2, T>0, \boldsymbol{u}_{0} \in \boldsymbol{V} \cap \boldsymbol{H}_{p}^{m}$ with $m \geq 3$ and $\boldsymbol{u}$ be the solution of (1.1). We assume that $\overline{\boldsymbol{u}}_{N}^{i}$ and $\boldsymbol{u}_{N}^{i}(i=1, \cdots, k-1)$ are computed with a proper initialization procedure such that

$$
\begin{aligned}
& \left\|\overline{\boldsymbol{u}}_{N}^{i}-\boldsymbol{u}\left(\cdot, t_{i}\right)\right\|_{1},\left\|\boldsymbol{u}_{N}^{i}-\boldsymbol{u}\left(t_{i}\right)\right\|_{1}=O\left(\delta t^{k}+N^{1-m}\right), \\
& \left\|\overline{\boldsymbol{u}}_{N}^{i}-\boldsymbol{u}\left(\cdot, t_{i}\right)\right\|_{2},\left\|\boldsymbol{u}_{N}^{i}-\boldsymbol{u}\left(t_{i}\right)\right\|_{2}=O\left(\delta t^{k}+N^{2-m}\right),
\end{aligned} \quad i=1,2,3,4,5 .
$$

Let $\overline{\boldsymbol{u}}_{N}^{n+1}$ and $\boldsymbol{u}_{N}^{n+1}$ be computed with the kth-order scheme $3.14(1 \leq k \leq 5)$, and

$$
\eta_{1}^{n+1}=1-\left(1-\xi^{n+1}\right)^{2}, \quad \eta_{k}^{n+1}=1-\left(1-\xi^{n+1}\right)^{k}(k=2,3,4,5) .
$$

Then for $n+1 \leq T / \delta t$ with $\delta t \leq \frac{1}{1+2^{k+2} C_{0}^{k+1}}$ and $N \geq 2^{k+2} C_{\Pi}^{k+1}+1$, we have

$$
\left\|\overline{\boldsymbol{u}}_{N}^{n}-\boldsymbol{u}\left(\cdot, t^{n}\right)\right\|_{1}^{2},\left\|\boldsymbol{u}_{N}^{n}-\boldsymbol{u}\left(\cdot, t^{n}\right)\right\|_{1}^{2} \leq C \delta t^{2 k}+C N^{2(1-m)}
$$

and

$$
\delta t \sum_{q=0}^{n}\left\|\overline{\boldsymbol{u}}_{N}^{q+1}-\boldsymbol{u}\left(\cdot, t^{q+1}\right)\right\|_{2}^{2}, \delta t \sum_{q=0}^{n}\left\|\boldsymbol{u}_{N}^{q+1}-\boldsymbol{u}\left(\cdot, t^{q+1}\right)\right\|_{2}^{2} \leq C \delta t^{2 k}+C N^{2(2-m)} .
$$

where the constants $C_{0}, C_{\Pi}$ and $C$ are dependent on $T, \Omega$, the $k \times k$ matrix $G=\left(g_{i j}\right)$ in Lemma 4 and the exact solution $\boldsymbol{u}$, but are independent of $\delta t$ and $N$. 
Proof. It is shown in [33] that in the periodic case, $\boldsymbol{u}_{0} \in \boldsymbol{H}_{p}^{m}$ implies that $\boldsymbol{u}(\cdot, t) \in \boldsymbol{H}_{p}^{m}$ for all $t \leq T$, and furthermore, it is shown in [11 that $\boldsymbol{u}$ has Gevrey class regularity. In particular, we have

$$
\boldsymbol{u} \in C\left([0, T] ; \boldsymbol{H}_{p}^{m}\right), m \geq 3, \frac{\partial^{j} \boldsymbol{u}}{\partial t^{j}} \in L^{2}\left(0, T ; \boldsymbol{H}_{p}^{2}\right) 1 \leq j \leq k, \frac{\partial^{k+1} \boldsymbol{u}}{\partial t^{k+1}} \in L^{2}\left(0, T ; L_{0}^{2}\right) .
$$

To simplify the presentation, we assume $\overline{\boldsymbol{u}}_{N}^{i}=\boldsymbol{u}_{N}^{i}=\Pi_{N} \boldsymbol{u}\left(t_{i}\right)$ and $r^{i}=E_{1}\left[\boldsymbol{u}_{N}^{i}\right]$ for $i=1, \cdots, k-1$ so that (4.1) is obviously satisfied.

The main task is to prove by induction,

$$
\left|1-\xi^{q}\right| \leq C_{0} \delta t+C_{\Pi} N^{2-m}, \forall q \leq T / \delta t,
$$

where the constant $C_{0}$ and $C_{\Pi}$ will be defined in the induction process below.

Under the assumption, 4.3 certainly holds for $q=0$. Now suppose we have

$$
\left|1-\xi^{q}\right| \leq C_{0} \delta t+C_{\Pi} N^{2-m}, \forall q \leq n,
$$

we shall prove below

$$
\left|1-\xi^{n+1}\right| \leq C_{0} \delta t+C_{\Pi} N^{2-m} .
$$

We shall first consider $k=2,3,4,5$, and point out the necessary modifications for the case $k=1$ later.

Step 1: Bounds for $\nabla \overline{\boldsymbol{u}}_{N}^{q}, \Delta \overline{\boldsymbol{u}}_{N}^{q}$ and $\Delta \boldsymbol{u}_{N}^{q}, \forall q \leq n$. We first recall the inequality

$$
(a+b)^{k} \leq 2^{k}\left(a^{k}+b^{k}\right), \quad \forall a, b>0, k \geq 1 .
$$

Under the assumption (4.4), if we choose $\delta t$ small enough and $N$ large enough such that

$$
\delta t \leq \min \left\{\frac{1}{2^{k+2} C_{0}^{k}}, 1\right\}, \quad N \geq \max \left\{2^{k+2} C_{\Pi}^{k}, 1\right\},
$$

we have

$$
1-\left(\frac{1}{2^{k+2} C_{0}^{k-1}}+\frac{N^{3-m}}{2^{k+2} C_{\Pi}^{k-1}}\right) \leq\left|\xi^{q}\right| \leq 1+\left(\frac{1}{2^{k+2} C_{0}^{k-1}}+\frac{N^{3-m}}{2^{k+2} C_{\Pi}^{k-1}}\right), \forall q \leq n,
$$

and

and

$$
\left(1-\xi^{q}\right)^{k} \leq \frac{\delta t^{k-1}}{4}+\frac{N^{k(2-m)+1}}{4}, \forall q \leq n,
$$

$$
\frac{1}{2}<1-\left(\frac{\delta t^{k-1}}{4}+\frac{N^{k(2-m)+1}}{4}\right) \leq\left|\eta_{k}^{q}\right| \leq 1+\frac{\delta t^{k-1}}{4}+\frac{N^{k(2-m)+1}}{4}<2, \forall q \leq n .
$$

Then it follows from the above and (3.17) that

$$
\left\|\overline{\boldsymbol{u}}_{N}^{q}\right\|_{1} \leq 2 M_{k}, \forall q \leq n .
$$

Moveover, 3.16 and $m \geq 3$ imply that

$$
\nu \delta t \sum_{q=1}^{n}\left\|\Delta \overline{\boldsymbol{u}}_{N}^{q}\right\|^{2} \leq \frac{2 r^{0}}{\left|\xi^{q}\right|} \leq 4 r^{0}, C_{0} \geq 1, C_{\Pi} \geq 1 .
$$

and

$$
\nu \delta t \sum_{q=1}^{n}\left\|\Delta \boldsymbol{u}_{N}^{q}\right\|^{2} \leq 16 r^{0}, C_{0} \geq 1, C_{\Pi} \geq 1 .
$$

Step 2: Estimates for $\nabla \overline{\boldsymbol{e}}_{N}^{n+1}$ and $\Delta \overline{\boldsymbol{e}}_{N}^{n+1}$. By the assumptions on the exact solution $\boldsymbol{u}$ and 4.9 , we can choose $C$ large enough such that

$$
\|\boldsymbol{u}(t)\|_{H^{2}}^{2} \leq C, \forall t \leq T,\left\|\overline{\boldsymbol{u}}_{N}^{q}\right\|_{1} \leq C, \forall q \leq n .
$$

From 3.14a), we can write down the error equation as

$$
\left(\alpha_{k} \overline{\boldsymbol{e}}^{q+1}-A_{k}\left(\overline{\boldsymbol{e}}^{q}\right), v_{N}\right)+\delta t \nu\left(\nabla \overline{\boldsymbol{e}}^{q+1}, \nabla v_{N}\right)=\left(R_{k}^{q}, v_{N}\right)+\delta t\left(Q_{k}^{q}, v_{N}\right), \quad \forall v_{N} \in S_{N},
$$


where $Q_{k}^{q}$ and $R_{k}^{q}$ are given by

$$
\left.Q_{k}^{q}=-\mathbf{A}\left(\left(B_{k} \boldsymbol{u}^{q}\right) \cdot \nabla\right) B_{k}\left(\boldsymbol{u}^{q}\right)\right)+\mathbf{A}\left(\boldsymbol{u}\left(t^{q+1}\right) \cdot \nabla \boldsymbol{u}\left(t^{q+1}\right)\right),
$$

and

$$
\begin{aligned}
R_{k}^{q} & =-\alpha_{k} \boldsymbol{u}\left(t^{q+1}\right)+A_{k}\left(\boldsymbol{u}\left(t^{q}\right)\right)+\delta t \boldsymbol{u}_{t}\left(t^{q+1}\right) \\
& =\sum_{i=1}^{k} a_{i} \int_{t^{q+1-i}}^{t^{q+1}}\left(t^{q+1-i}-s\right)^{k} \frac{\partial^{k+1} \boldsymbol{u}}{\partial t^{k+1}}(s) d s,
\end{aligned}
$$

with $a_{i}$ being some fixed and bounded constants determined by the truncation errors, for example, in the case $k=3$, we have

$$
R_{3}^{q}=-3 \int_{t^{q}}^{t^{q+1}}\left(t^{q}-s\right)^{3} \frac{\partial^{4} \boldsymbol{u}}{\partial t^{4}}(s) d s+\frac{3}{2} \int_{t^{q-1}}^{t^{n+1}}\left(t^{q-1}-s\right)^{3} \frac{\partial^{4} \boldsymbol{u}}{\partial t^{4}}(s) d s-\frac{1}{3} \int_{t^{q-2}}^{t^{n+1}}\left(t^{q-2}-s\right)^{3} \frac{\partial^{4} \boldsymbol{u}}{\partial t^{4}}(s) d s .
$$

Let $v_{N}=-\Delta \overline{\boldsymbol{e}}_{N}^{q+1}+\tau_{k} \Delta \overline{\boldsymbol{e}}_{N}^{q}$ in 4.13 , it follows from Lemma 4 and 3.13 that

$$
\begin{aligned}
\sum_{i, j=1}^{k} g_{i j} & \left(\nabla \overline{\boldsymbol{e}}_{N}^{q+1+i-k}, \nabla \overline{\boldsymbol{e}}_{N}^{q+1+j-k}\right)-\sum_{i, j=1}^{k} g_{i j}\left(\nabla \overline{\boldsymbol{e}}_{N}^{q+i-k}, \nabla \overline{\boldsymbol{e}}_{N}^{q+j-k}\right) \\
& +\left\|\sum_{i=0}^{k} \delta_{i} \nabla \overline{\boldsymbol{e}}_{N}^{q+1+i-k}\right\|^{2}+\delta t \nu\left\|\Delta \overline{\boldsymbol{e}}_{N}^{q+1}\right\|^{2} \\
& =\delta t \nu\left(\Delta \overline{\boldsymbol{e}}_{N}^{q+1}, \tau_{k} \Delta \overline{\boldsymbol{e}}_{N}^{q}\right)+\left(R_{k}^{q},-\Delta \overline{\boldsymbol{e}}_{N}^{q+1}+\tau_{k} \Delta \overline{\boldsymbol{e}}_{N}^{q}\right)+\delta t\left(Q_{k}^{n},-\Delta \overline{\boldsymbol{e}}_{N}^{q+1}+\tau_{k} \Delta \overline{\boldsymbol{e}}_{N}^{q}\right) .
\end{aligned}
$$

Next, we bound the righthand side of (4.16). It follows from 4.15) that

$$
\left\|R_{k}^{q}\right\|^{2} \leq C \delta t^{2 k+1} \int_{t^{q+1-k}}^{t^{q+1}}\left\|\frac{\partial^{k+1} \boldsymbol{u}}{\partial t^{k+1}}(s)\right\|^{2} d s
$$

Therefore,

$$
\begin{aligned}
\mid\left(R_{k}^{q},\right. & \left.-\Delta \overline{\boldsymbol{e}}_{N}^{q+1}+\tau_{k} \Delta \overline{\boldsymbol{e}}_{N}^{q}\right) \mid \leq \frac{C(\varepsilon)}{\delta t}\left\|R_{k}^{q}\right\|^{2}+\delta t \varepsilon\left\|-\Delta \overline{\boldsymbol{e}}_{N}^{q+1}+\tau_{k} \Delta \overline{\boldsymbol{e}}_{N}^{q}\right\|^{2}, \\
& \leq \frac{C(\varepsilon)}{\delta t}\left\|R_{k}^{q}\right\|^{2}+2 \delta t \varepsilon\left\|\Delta \overline{\boldsymbol{e}}_{N}^{q+1}\right\|^{2}+2 \delta t \varepsilon\left\|\Delta \overline{\boldsymbol{e}}_{N}^{q}\right\|^{2} \\
& \leq 2 \delta t \varepsilon\left\|\Delta \overline{\boldsymbol{e}}_{N}^{q+1}\right\|^{2}+2 \delta t \varepsilon\left\|\Delta \overline{\boldsymbol{e}}_{N}^{q}\right\|^{2}+C(\varepsilon) \delta t^{2 k} \int_{t^{q+1-k}}^{t^{q+1}}\left\|\frac{\partial^{k+1} \boldsymbol{u}}{\partial t^{k+1}}(s)\right\|^{2} d s .
\end{aligned}
$$

For the term with $Q_{k}^{q}$, we split it as

$$
\begin{aligned}
\left(Q_{k}^{n},-\Delta \overline{\boldsymbol{e}}_{N}^{q+1}+\tau_{k} \Delta \boldsymbol{e}_{N}^{q}\right) & =\left(\mathbf{A}\left(\left[\boldsymbol{u}\left(t^{q+1}\right)-B_{k}\left(\boldsymbol{u}^{q}\right)\right] \cdot \nabla \boldsymbol{u}\left(t^{q+1}\right)\right),-\Delta \overline{\boldsymbol{e}}_{N}^{q+1}+\tau_{k} \Delta \boldsymbol{e}_{N}^{q}\right) \\
& +\left(\mathbf{A}\left(B_{k}\left(\boldsymbol{u}^{q}\right) \cdot \nabla\left[\boldsymbol{u}\left(t^{q+1}\right)-B_{k}\left(\boldsymbol{u}\left(t^{q}\right)\right)\right]\right),-\Delta \overline{\boldsymbol{e}}_{N}^{q+1}+\tau_{k} \Delta \overline{\boldsymbol{e}}_{N}^{q}\right) \\
& -\left(\mathbf{A}\left(B_{k}\left(\boldsymbol{e}^{q}\right) \cdot \nabla B_{k}\left(\boldsymbol{e}^{q}\right)\right),-\Delta \overline{\boldsymbol{e}}_{N}^{q+1}+\tau_{k} \Delta \overline{\boldsymbol{e}}_{N}^{q}\right) \\
& -\left(\mathbf{A}\left(B_{k}\left(\boldsymbol{u}\left(t^{q}\right)\right) \cdot \nabla B_{k}\left(\boldsymbol{e}^{q}\right)\right),-\Delta \overline{\boldsymbol{e}}_{N}^{q+1}+\tau_{k} \Delta \overline{\boldsymbol{e}}_{N}^{q}\right) .
\end{aligned}
$$


We bound the terms on the right hand side of 4.19 ) with the help of 2.7$), 2.9)$ and 4.12):

$$
\begin{aligned}
& \left(\mathbf{A}\left(\left[\boldsymbol{u}\left(t^{q+1}\right)-B_{k}\left(\boldsymbol{u}^{q}\right)\right] \cdot \nabla \boldsymbol{u}\left(t^{q+1}\right)\right),-\Delta \overline{\boldsymbol{e}}_{N}^{q+1}+\tau_{k} \Delta \overline{\boldsymbol{e}}_{N}^{q}\right) \\
& \leq C\left\|\boldsymbol{u}\left(t^{q+1}\right)-B_{k}\left(\boldsymbol{u}^{q}\right)\right\|_{1}\left\|\boldsymbol{u}\left(t^{q+1}\right)\right\|_{2}\left\|-\Delta \overline{\boldsymbol{e}}_{N}^{q+1}+\tau_{k} \Delta \overline{\boldsymbol{e}}_{N}^{q}\right\| \\
& \leq C(\varepsilon)\left\|\boldsymbol{u}\left(t^{q+1}\right)-B_{k}\left(\boldsymbol{u}^{q}\right)\right\|_{1}^{2}\left\|\boldsymbol{u}\left(t^{q+1}\right)\right\|_{2}^{2}+\varepsilon\left\|-\Delta \overline{\boldsymbol{e}}_{N}^{q+1}+\tau_{k} \Delta \overline{\boldsymbol{e}}_{N}^{q}\right\|^{2} \\
& \leq C(\varepsilon)\left\|\boldsymbol{u}\left(t^{q+1}\right)-B_{k}\left(\boldsymbol{u}\left(t^{q}\right)\right)\right\|_{1}^{2}\left\|\boldsymbol{u}\left(t^{q+1}\right)\right\|_{2}^{2}+C(\varepsilon)\left\|B_{k}\left(\boldsymbol{e}^{q}\right)\right\|_{1}^{2}\left\|\boldsymbol{u}\left(t^{q+1}\right)\right\|_{2}^{2}+\varepsilon\left\|-\Delta \overline{\boldsymbol{e}}_{N}^{q+1}+\tau_{k} \Delta \overline{\boldsymbol{e}}_{N}^{q}\right\|^{2} \\
& \leq C(\varepsilon)\left\|\sum_{i=1}^{k} b_{i} \int_{t^{q+1-i}}^{t^{q+1}}\left(t^{q+1-i}-s\right)^{k-1} \frac{\partial^{k} \boldsymbol{u}}{\partial t^{k}}(s) d s\right\|_{1}^{2}+C(\varepsilon)\left\|B_{k}\left(\boldsymbol{e}^{q}\right)\right\|_{1}^{2}+2 \varepsilon\left\|\Delta \overline{\boldsymbol{e}}_{N}^{q+1}\right\|^{2}+2 \varepsilon\left\|\Delta \overline{\boldsymbol{e}}_{N}^{q}\right\|^{2} \\
& \leq C(\varepsilon) \delta t^{2 k-1} \int_{t^{q+1-k}}^{t^{q+1}}\left\|\frac{\partial^{k} \boldsymbol{u}}{\partial t^{k}}(s)\right\|_{1}^{2} d s+C(\varepsilon)\left\|B_{k}\left(\boldsymbol{e}^{q}\right)\right\|_{1}^{2}+2 \varepsilon\left\|\Delta \overline{\boldsymbol{e}}_{N}^{q+1}\right\|^{2}+2 \varepsilon\left\|\Delta \overline{\boldsymbol{e}}_{N}^{q}\right\|^{2},
\end{aligned}
$$

where $b_{i}$ are some fixed and bounded constants determined by the truncation error. For example, in the case $k=3$, we have

$$
\begin{aligned}
B_{3}\left(\boldsymbol{u}\left(t^{q}\right)\right)-\boldsymbol{u}\left(t^{q+1}\right) & =-\frac{3}{2} \int_{t^{q}}^{t^{q+1}}\left(t^{q}-s\right)^{2} \frac{\partial^{3} \boldsymbol{u}}{\partial t^{3}}(s) d s+\frac{3}{2} \int_{t^{q-1}}^{t^{q+1}}\left(t^{q-1}-s\right)^{2} \frac{\partial^{3} \boldsymbol{u}}{\partial t^{3}} d s \\
& -\frac{1}{2} \int_{t^{q-2}}^{t^{q+1}}\left(t^{q-2}-s\right)^{2} \frac{\partial^{3} \boldsymbol{u}}{\partial t^{3}} d s .
\end{aligned}
$$

For the other terms in the righthand side of 4.19], we have

$$
\begin{aligned}
\mid\left(\mathbf { A } \left(B_{k}\left(\boldsymbol{u}^{q}\right)\right.\right. & \left.\left.\cdot \nabla\left[\boldsymbol{u}\left(t^{q+1}\right)-B_{k}\left(\boldsymbol{u}\left(t^{q}\right)\right)\right]\right),-\Delta \overline{\boldsymbol{e}}_{N}^{q+1}+\tau_{k} \Delta \overline{\boldsymbol{e}}_{N}^{q}\right) \mid \\
& \leq C\left\|B_{k}\left(\boldsymbol{u}^{q}\right)\right\|_{1}\left\|\boldsymbol{u}\left(t^{q+1}\right)-B_{k}\left(\boldsymbol{u}\left(t^{q}\right)\right)\right\|_{2}\left\|-\Delta \overline{\boldsymbol{e}}_{N}^{q+1}+\tau_{k} \Delta \overline{\boldsymbol{e}}_{N}^{q}\right\| \\
& \leq C(\varepsilon)\left\|B_{k}\left(\boldsymbol{u}^{q}\right)\right\|_{1}^{2}\left\|\boldsymbol{u}\left(t^{q+1}\right)-B_{k}\left(\boldsymbol{u}\left(t^{q}\right)\right)\right\|_{2}^{2}+\varepsilon\left\|-\Delta \overline{\boldsymbol{e}}_{N}^{q+1}+\tau_{k} \Delta \overline{\boldsymbol{e}}_{N}^{q}\right\|^{2} \\
& \leq C(\varepsilon) \delta t^{2 k-1} \int_{t^{q+1-k}}^{t^{q+1}}\left\|\frac{\partial^{k} \boldsymbol{u}}{\partial t^{k}}(s)\right\|_{2}^{2} d s+2 \varepsilon\left\|\Delta \overline{\boldsymbol{e}}_{N}^{q+1}\right\|^{2}+2 \varepsilon\left\|\Delta \overline{\boldsymbol{e}}_{N}^{q}\right\|^{2}
\end{aligned}
$$

Since $d=2$, we can use (2.7) to obtain

$$
\begin{aligned}
& \left|\left(\mathbf{A}\left(B_{k}\left(\boldsymbol{e}^{q}\right) \cdot \nabla B_{k}\left(\boldsymbol{e}^{q}\right)\right),-\Delta \overline{\boldsymbol{e}}_{N}^{q+1}+\tau_{k} \Delta \overline{\boldsymbol{e}}_{N}^{q}\right)\right| \\
& \quad \leq C\left\|B_{k}\left(\overline{\boldsymbol{e}}^{q}\right)\right\|_{1}^{1 / 2}\left\|B_{k}\left(\overline{\boldsymbol{e}}^{q}\right)\right\|^{1 / 2}\left\|B_{k}\left(\overline{\boldsymbol{e}}^{q}\right)\right\|_{2}^{1 / 2}\left\|B_{k}\left(\overline{\boldsymbol{e}}^{q}\right)\right\|_{1}^{1 / 2}\left\|-\Delta \overline{\boldsymbol{e}}_{N}^{q+1}+\tau_{k} \Delta \overline{\boldsymbol{e}}_{N}^{q}\right\| \\
& \quad \leq C\left\|B_{k}\left(\boldsymbol{e}^{q}\right)\right\|_{1}\left\|B_{k}\left(\boldsymbol{e}^{q}\right)\right\|_{2}\left\|-\Delta \overline{\boldsymbol{e}}_{N}^{q+1}+\tau_{k} \Delta \overline{\boldsymbol{e}}_{N}^{q}\right\| \quad \text { (true in 2d and 3d) } \\
& \quad \leq C(\varepsilon)\left\|B_{k}\left(\boldsymbol{e}^{q}\right)\right\|_{1}^{2}\left\|B_{k}\left(\boldsymbol{e}^{q}\right)\right\|_{2}^{2}+\varepsilon\left\|-\Delta \overline{\boldsymbol{e}}_{N}^{q+1}+\tau_{k} \Delta \overline{\boldsymbol{e}}_{N}^{q}\right\|^{2} \\
& \quad \leq C(\varepsilon)\left\|B_{k}\left(\boldsymbol{e}^{q}\right)\right\|_{1}^{2}\left\|B_{k}\left(\boldsymbol{e}^{q}\right)\right\|_{2}^{2}+2 \varepsilon\left\|\Delta \overline{\boldsymbol{e}}_{N}^{q+1}\right\|^{2}+2 \varepsilon\left\|\Delta \overline{\boldsymbol{e}}_{N}^{q}\right\|^{2}
\end{aligned}
$$

Thanks to 2.9 , we have

$$
\begin{aligned}
& \left|\left(\mathbf{A}\left(B_{k}\left(\boldsymbol{u}\left(t^{q}\right)\right) \cdot \nabla B_{k}\left(\overline{\boldsymbol{e}}^{q}\right)\right),-\Delta \overline{\boldsymbol{e}}_{N}^{q+1}+\tau_{k} \Delta \overline{\boldsymbol{e}}_{N}^{q}\right)\right| \\
& \quad \leq C\left\|B_{k}\left(\boldsymbol{u}\left(t^{q}\right)\right)\right\|_{2}\left\|B_{k}\left(\boldsymbol{e}^{q}\right)\right\|_{1}\left\|-\Delta \overline{\boldsymbol{e}}_{N}^{q+1}+\tau_{k} \Delta \overline{\boldsymbol{e}}_{N}^{q}\right\| \\
& \quad \leq C(\varepsilon)\left\|B_{k}\left(\boldsymbol{u}\left(t^{q}\right)\right)\right\|_{2}^{2}\left\|B_{k}\left(\boldsymbol{e}^{q}\right)\right\|_{1}^{2}+\varepsilon\left\|-\Delta \overline{\boldsymbol{e}}_{N}^{q+1}+\tau_{k} \Delta \overline{\boldsymbol{e}}_{N}^{q}\right\|^{2} \\
& \quad \leq C(\varepsilon)\left\|B_{k}\left(\boldsymbol{e}^{q}\right)\right\|_{1}^{2}+2 \varepsilon\left\|\Delta \overline{\boldsymbol{e}}_{N}^{q+1}\right\|^{2}+2 \varepsilon\left\|\Delta \overline{\boldsymbol{e}}_{N}^{q}\right\|^{2} .
\end{aligned}
$$

On the other hand, we derive from 4.6 and 4.4 that

$$
\left|\eta_{k}^{q}-1\right| \leq 2^{k} C_{0}^{k} \delta t^{k}+2^{k} C_{\Pi}^{k} N^{k(2-m)}, \quad \forall q \leq n .
$$


Note that $\boldsymbol{u}_{N}^{q}=\eta_{k}^{q} \overline{\boldsymbol{u}}_{N}^{q}$, we can estimate $\left\|B_{k}\left(\boldsymbol{e}^{q}\right)\right\|_{1}^{2}$ by

$$
\begin{aligned}
\left\|B_{k}\left(\boldsymbol{e}^{q}\right)\right\|_{1}^{2} & =\left\|B_{k}\left(\boldsymbol{u}_{N}^{q}-\overline{\boldsymbol{u}}_{N}^{q}\right)+B_{k}\left(\overline{\boldsymbol{e}}_{N}^{q}\right)+B_{k}\left(\boldsymbol{e}_{\Pi}^{q}\right)\right\|_{1}^{2} \\
& \leq C C_{0}^{2 k} \delta t^{2 k}+C C_{\Pi}^{2 k} N^{2 k(2-m)}+C\left\|B_{k}\left(\overline{\boldsymbol{e}}_{N}^{q}\right)\right\|_{1}^{2}+C\left\|\boldsymbol{u}\left(t^{q}\right)\right\|_{m}^{2} N^{2-2 m} .
\end{aligned}
$$

Combining (4.16)-(4.24) and dropping some unnecessary terms, we arrive at

$$
\begin{aligned}
\sum_{i, j=1}^{k} g_{i j}( & \left.\nabla \overline{\boldsymbol{e}}_{N}^{q+1+i-k}, \nabla \overline{\boldsymbol{e}}_{N}^{q+1+j-k}\right)-\sum_{i, j=1}^{k} g_{i j}\left(\nabla \overline{\boldsymbol{e}}_{N}^{q+i-k}, \nabla \overline{\boldsymbol{e}}_{N}^{q+j-k}\right)+\delta t\left(\frac{\nu}{2}-10 \varepsilon\right)\left\|\Delta \overline{\boldsymbol{e}}_{N}^{q+1}\right\|^{2} \\
\leq & \delta t\left(\frac{\nu \tau_{k}^{2}}{2}+10 \varepsilon\right)\left\|\Delta \overline{\boldsymbol{e}}_{N}^{q}\right\|^{2}+C(\varepsilon) \delta t\left\|B_{k}\left(\overline{\boldsymbol{e}}_{N}^{q}\right)\right\|_{1}^{2}+C(\varepsilon) \delta t\left\|B_{k}\left(\overline{\boldsymbol{e}}_{N}^{q}\right)\right\|_{1}^{2}\left\|B_{k}\left(\boldsymbol{e}_{N}^{q}\right)\right\|_{2}^{2} \\
& +C(\varepsilon) \delta t^{2 k} \int_{t^{q+1-k}}^{t^{q+1}}\left(\left\|\frac{\partial^{k} \boldsymbol{u}}{\partial t^{k}}(s)\right\|_{2}^{2}+\left\|\frac{\partial^{k+1} \boldsymbol{u}}{\partial t^{k+1}}(s)\right\|^{2}\right) d s \\
& +C(\varepsilon) C_{0}^{2 k} \delta t^{2 k+1}\left(1+\left\|B_{k}\left(\boldsymbol{e}^{q}\right)\right\|_{2}^{2}\right)+\delta t C(\varepsilon) C_{\Pi}^{2 k} N^{2 k(2-m)}\left(1+\left\|B_{k}\left(\boldsymbol{e}^{q}\right)\right\|_{2}^{2}\right) \\
& +\delta t C(\varepsilon)\left\|\boldsymbol{u}\left(t^{q}\right)\right\|_{m}^{2} N^{2-2 m}\left(1+\left\|B_{k}\left(\boldsymbol{e}^{q}\right)\right\|_{2}^{2}\right) .
\end{aligned}
$$

Since $\tau_{k}<1$, we can choose $\varepsilon$ small enough such that

$$
\frac{\nu}{2}-10 \varepsilon>\frac{\nu \tau_{k}^{2}}{2}+10 \varepsilon+\frac{\nu\left(1-\tau_{k}^{2}\right)}{4}
$$

and then taking the sum of 4.25) on $q$ from $k-1$ to $n$, noting that $G=\left(g_{i j}\right)$ is a symmetric positive definite matrix with minimum eigenvalue $\lambda_{G}$, we obtain:

$$
\begin{aligned}
\lambda_{G}\left\|\nabla \overline{\boldsymbol{e}}_{N}^{n+1}\right\|^{2} & +\frac{\delta t \nu\left(1-\tau_{k}^{2}\right)}{4} \sum_{q=0}^{n+1}\left\|\Delta \overline{\boldsymbol{e}}_{N}^{q}\right\|^{2} \\
& \leq \sum_{i, j=1}^{k} g_{i j}\left(\nabla \overline{\boldsymbol{e}}_{N}^{n+1+i-k}, \nabla \overline{\boldsymbol{e}}_{N}^{n+1+j-k}\right)+\frac{\delta t \nu\left(1-\tau_{k}^{2}\right)}{4} \sum_{q=0}^{n+1}\left\|\Delta \overline{\boldsymbol{e}}_{N}^{q}\right\|^{2} \\
& \leq C \delta t \sum_{q=0}^{n}\left\|\overline{\boldsymbol{e}}_{N}^{q}\right\|_{1}^{2}\left(\left\|B_{k}\left(\boldsymbol{e}^{q}\right)\right\|_{2}^{2}+1\right) \\
& +C \delta t^{2 k}\left(\int_{0}^{T}\left(\left\|\frac{\partial^{k} \boldsymbol{u}}{\partial t^{k}}(s)\right\|_{2}^{2}+\left\|\frac{\partial^{k+1} \boldsymbol{u}}{\partial t^{k+1}}(s)\right\|^{2}\right) d s+C_{0}^{2 k}\left(T+\delta t \sum_{q=0}^{n}\left\|B_{k}\left(\boldsymbol{e}^{q}\right)\right\|_{2}^{2}\right)\right) \\
& +C\left(C_{\Pi}^{2 k} N^{2 k(2-m)}+N^{2-2 m}\right)\left(T+\delta t \sum_{q=0}^{n}\left\|B_{k}\left(\boldsymbol{e}^{q}\right)\right\|_{2}^{2}\right) .
\end{aligned}
$$

Noting that 4.11 and 4.12 imply $\delta t \sum_{q=0}^{n}\left\|B_{k}\left(\boldsymbol{e}^{q}\right)\right\|_{2}^{2}<C_{H^{2}}$ for some constant $C_{H^{2}}$ depends only on the exact solution $\boldsymbol{u}$. Applying the discrete Gronwall Lemma 3 to 4.27), we obtain

$$
\begin{aligned}
& \left\|\overline{\boldsymbol{e}}_{N}^{n+1}\right\|_{1}^{2}+\delta t \sum_{q=0}^{n+1}\left\|\overline{\boldsymbol{e}}_{N}^{q}\right\|_{2}^{2} \\
& \left.\leq C \exp \left(C_{H^{2}}+1\right)\right) \delta t^{2 k} \int_{0}^{T}\left(\left\|\frac{\partial^{k} \boldsymbol{u}}{\partial t^{k}}(s)\right\|_{2}^{2}+\left\|\frac{\partial^{k+1} \boldsymbol{u}}{\partial t^{k+1}}(s)\right\|^{2}\right) d s \\
& \left.+C \exp \left(C_{H^{2}}+1\right)\right)\left(\delta t^{2 k} C_{0}^{2 k}+C_{\Pi}^{2 k} N^{2 k(2-m)}+N^{2-2 m}\right)\left(T+C_{H^{2}}\right) \\
& \leq C_{1}\left(1+C_{0}^{2 k}\right) \delta t^{2 k}+C_{1}\left(C_{\Pi}^{2 k} N^{2 k(2-m)}+N^{2-2 m}\right),
\end{aligned}
$$

where $C_{1}$ is independent of $\delta t, C_{0}, C_{\Pi}$, and can be defined as

$$
C_{1}:=C \exp \left(C_{H^{2}}+1\right) \max \left(\int_{0}^{T}\left(\left\|\frac{\partial^{k} \boldsymbol{u}}{\partial t^{k}}(s)\right\|_{2}^{2}+\left\|\frac{\partial^{k+1} \boldsymbol{u}}{\partial t^{k+1}}(s)\right\|^{2}\right) d s, 1, T+C_{H^{2}}\right) .
$$


Therefore, 4.28 implies

$$
\left\|\overline{\boldsymbol{e}}_{N}^{n+1}\right\|_{1}^{2}, \delta t \sum_{q=0}^{n+1}\left\|\overline{\boldsymbol{e}}_{N}^{q}\right\|_{2}^{2} \leq C_{1}\left(1+C_{0}^{2 k}\right) \delta t^{2 k}+C_{1}\left(C_{\Pi}^{2 k} N^{2 k(2-m)}+N^{2-2 m}\right) .
$$

Since $\overline{\boldsymbol{e}}^{q}=\overline{\boldsymbol{e}}_{N}^{q}+\overline{\boldsymbol{e}}_{\Pi}^{q}$, it follows from the triangle inequality that

$$
\left\|\overline{\boldsymbol{e}}^{n+1}\right\|_{1}^{2} \leq C_{1}\left(1+C_{0}^{2 k}\right) \delta t^{2 k}+C_{1}\left(C_{\Pi}^{2 k} N^{2 k(2-m)}+N^{2-2 m}\right)+C N^{2(1-m)},
$$

and

$$
\delta t \sum_{q=0}^{n+1}\left\|\bar{e}^{q}\right\|_{2}^{2} \leq C_{1}\left(1+C_{0}^{2 k}\right) \delta t^{2 k}+C_{1}\left(C_{\Pi}^{2 k} N^{2 k(2-m)}+N^{2-2 m}\right)+C N^{2(2-m)} .
$$

Combining 4.12, 4.31 and 4.32, we find that, under the condition 4.7) and $m \geq 3$, we have

$$
\begin{aligned}
\left\|\overline{\boldsymbol{u}}_{N}^{n+1}\right\|_{1}^{2}, \delta t \sum_{q=0}^{n+1}\left\|\overline{\boldsymbol{u}}_{N}^{q}\right\|_{2}^{2} & \leq C_{1}\left(1+C_{0}^{2 k} \frac{1}{2^{2 k(k+2)} C_{0}^{2 k^{2}}}\right)+C_{1}\left(C_{\Pi}^{2 k} 2^{-4 k(k+1)} C_{\Pi}^{-4 k^{2}}+1\right)+C \\
& \leq 4 C_{1}+C:=\bar{C} .
\end{aligned}
$$

Step 3: Estimate for $\left|1-\xi^{n+1}\right|$. It follows from 3.14b that the equation for $\left\{s^{j}\right\}$ can be written as

$$
s^{q+1}-s^{q}=\delta t \nu\left(\left\|\Delta \boldsymbol{u}\left(t^{q+1}\right)\right\|^{2}-\frac{r^{q+1}}{E\left(\overline{\boldsymbol{u}}_{N}^{q+1}\right)+1}\left\|\Delta \overline{\boldsymbol{u}}_{N}^{q+1}\right\|^{2}\right)+T_{q}, \quad \forall q \leq n,
$$

where $T_{q}$ is the truncation error

$$
T_{q}=r\left(t^{q}\right)-r\left(t^{q+1}\right)+\delta t r_{t}\left(t^{q+1}\right)=\int_{t^{q}}^{t^{q+1}}\left(s-t^{q}\right) r_{t t}(s) d s .
$$

Taking the sum of 4.34 for $q$ from 0 to $n$, and noting that $s^{0}=0$, we have

$$
s^{n+1}=\delta t \nu \sum_{q=0}^{n}\left(\left\|\Delta \boldsymbol{u}\left(t^{q+1}\right)\right\|^{2}-\frac{r^{q+1}}{E\left(\overline{\boldsymbol{u}}_{N}^{q+1}\right)+1}\left\|\Delta \overline{\boldsymbol{u}}_{N}^{q+1}\right\|^{2}\right)+\sum_{q=0}^{n} T_{q},
$$

We bound the righthand side of 4.36 as follows. By direct calculation, we have

$$
r_{t t}=\int_{\Omega}\left((\nabla \boldsymbol{u})_{t}^{2}+\nabla \boldsymbol{u}(\nabla \boldsymbol{u})_{t t}\right) d x
$$

then from 4.35, we have

$$
\left|T_{q}\right| \leq C \delta t \int_{t^{q}}^{t^{q+1}}\left|r_{t t}\right| d s \leq C \delta t \int_{t^{q}}^{t^{q+1}}\left(\left\|\boldsymbol{u}_{t}\right\|_{1}^{2}+\left\|\boldsymbol{u}_{t t}\right\|_{1}^{2}\right) d s, \forall q \leq n .
$$

By triangular inequality,

$$
\begin{aligned}
& \left|\left\|\Delta \boldsymbol{u}\left(t^{q+1}\right)\right\|^{2}-\frac{r^{q+1}}{E\left(\overline{\boldsymbol{u}}_{N}^{q+1}\right)+1}\left\|\Delta \overline{\boldsymbol{u}}_{N}^{q+1}\right\|^{2}\right| \\
& \quad \leq\left\|\Delta \boldsymbol{u}\left(t^{q+1}\right)\right\|^{2}\left|1-\frac{r^{q+1}}{E\left(\overline{\boldsymbol{u}}_{N}^{q+1}\right)+1}\right|+\frac{r^{q+1}}{E\left(\overline{\boldsymbol{u}}_{N}^{q+1}\right)+1}\left|\left\|\Delta \boldsymbol{u}\left(t^{q+1}\right)\right\|^{2}-\left\|\Delta \overline{\boldsymbol{u}}_{N}^{q+1}\right\|^{2}\right| \\
& \quad:=K_{1}^{q}+K_{2}^{q} .
\end{aligned}
$$


It follows from 4.12 and Theorem 1 that

$$
\begin{aligned}
K_{1}^{q} & \leq C\left|1-\frac{r^{q+1}}{E\left(\overline{\boldsymbol{u}}_{N}^{q+1}\right)+1}\right| \\
& =C\left|\frac{r\left(t^{q+1}\right)}{E\left[\boldsymbol{u}\left(t^{q+1}\right)\right]+1}-\frac{r^{q+1}}{E\left[\boldsymbol{u}\left(t^{q+1}\right)\right]+1}\right|+C\left|\frac{r^{q+1}}{E\left[\boldsymbol{u}\left(t^{q+1}\right)\right]+1}-\frac{r^{q+1}}{E\left(\overline{\boldsymbol{u}}_{N}^{q+1}\right)+1}\right| \\
& \leq C\left(\left|E\left[\boldsymbol{u}\left(t^{q+1}\right)\right]-E\left(\overline{\boldsymbol{u}}_{N}^{q+1}\right)\right|+\left|s^{q+1}\right|\right), \forall q \leq n,
\end{aligned}
$$

and it follows from 4.12 and Theorem 1 that

$$
\begin{aligned}
K_{2}^{q} & \leq C\left|\left\|\Delta \overline{\boldsymbol{u}}_{N}^{q+1}\right\|^{2}-\left\|\Delta \boldsymbol{u}\left(t^{q+1}\right)\right\|^{2}\right| \\
& \leq C\left\|\Delta \overline{\boldsymbol{u}}_{N}^{q+1}-\Delta \boldsymbol{u}\left(t^{q+1}\right)\right\|\left(\left\|\Delta \overline{\boldsymbol{u}}^{q+1}\right\|+\left\|\Delta \boldsymbol{u}\left(t^{q+1}\right)\right\|\right) \\
& \leq C\left\|\Delta \overline{\boldsymbol{u}}_{N}^{q+1}\right\|\left\|\Delta \overline{\boldsymbol{e}}^{q+1}\right\|+C\left\|\Delta \overline{\boldsymbol{e}}^{q+1}\right\|, \quad \forall q \leq n
\end{aligned}
$$

We derive from the definition of $E(\boldsymbol{u})$ that

$$
\left|E\left(\boldsymbol{u}\left(t^{q+1}\right)\right)-E\left(\overline{\boldsymbol{u}}_{N}^{q+1}\right)\right| \leq \frac{1}{2}\left(\left\|\nabla \boldsymbol{u}\left(t^{q+1}\right)\right\|+\left\|\nabla \overline{\boldsymbol{u}}_{N}^{q+1}\right\|\right)\left\|\nabla \boldsymbol{u}\left(t^{q+1}\right)-\nabla \overline{\boldsymbol{u}}_{N}^{q+1}\right\| \leq C\left\|\nabla \overline{\boldsymbol{e}}^{q+1}\right\| .
$$

It follows from 4.32, 4.33 and the Cauchy-Schwarz inequality that

$$
\begin{aligned}
& \delta t \sum_{q=0}^{n}\left\|\Delta \overline{\boldsymbol{u}}_{N}^{q+1}\right\|\left\|\Delta \overline{\boldsymbol{e}}^{q+1}\right\| \leq\left(\delta t \sum_{q=0}^{n}\left\|\Delta \overline{\boldsymbol{u}}^{q+1}\right\|^{2} \delta t \sum_{q=0}^{n}\left\|\Delta \overline{\boldsymbol{e}}^{q+1}\right\|^{2}\right)^{1 / 2} \\
& \leq C \sqrt{C_{1}\left(1+C_{0}^{2 k}\right) \delta t^{2 k}+C_{1}\left(C_{\Pi}^{2 k} N^{2 k(2-m)}+N^{2-2 m}\right)+N^{2(2-m)}} .
\end{aligned}
$$

Now, we are ready to estimate $s^{n+1}$. Combining the estimates obtained above, (4.36) leads to

$$
\begin{aligned}
\left|s^{n+1}\right| & \leq \delta t \nu \sum_{q=0}^{n}\left|\left\|\nabla \boldsymbol{u}\left(t^{q+1}\right)\right\|^{2}-\frac{r^{q+1}}{E\left(\overline{\boldsymbol{u}}^{q+1}\right)+1}\left\|\nabla \overline{\boldsymbol{u}}_{N}^{q+1}\right\|^{2}\right|+\sum_{q=0}^{n}\left|T^{q}\right| \\
& \leq C \delta t \sum_{q=0}^{n}\left|s^{q+1}\right|+C \delta t \sum_{q=0}^{n}\left\|\overline{\boldsymbol{e}}^{q+1}\right\|_{2}+C \delta t \sum_{q=0}^{n}\left\|\Delta \overline{\boldsymbol{u}}_{N}^{q+1}\right\|\left\|\Delta \overline{\boldsymbol{e}}^{q+1}\right\| \\
& +C \delta t \int_{0}^{t^{n+1}}\left(\left\|\boldsymbol{u}_{t}\right\|_{1}^{2}+\left\|\boldsymbol{u}_{t t}\right\|_{1}^{2}\right) d s \\
& \leq C \sqrt{C_{1}\left(1+C_{0}^{2 k}\right) \delta t^{2 k}+C_{1}\left(C_{\Pi}^{2 k} N^{2 k(2-m)}+N^{2-2 m}\right)+N^{2(2-m)}} \\
& +C \delta t \sum_{q=0}^{n}\left|s^{q+1}\right|+C \delta t .
\end{aligned}
$$

Finally, applying Lemma 2 on 4.43 with $\delta t<\frac{1}{2 C}$, we obtain the following estimate for $s^{n+1}$ :

$$
\begin{aligned}
\left|s^{n+1}\right| & \leq C \exp \left((1-\delta t C)^{-1}\right)\left(\sqrt{C_{1}\left(1+C_{0}^{2 k}\right) \delta t^{2 k}+C_{1}\left(C_{\Pi}^{2 k} N^{2 k(2-m)}+N^{2-2 m}\right)+N^{2(2-m)}}+\delta t\right) \\
& \leq C_{2}\left(\sqrt{C_{1}\left(1+C_{0}^{2 k}\right) \delta t^{2 k}+C_{1}\left(C_{\Pi}^{2 k} N^{2 k(2-m)}+N^{2-2 m}\right)+N^{2(2-m)}}+\delta t\right) \\
& \leq C_{2} \delta t^{k} \sqrt{C_{1}\left(1+C_{0}^{2 k}\right)}+C_{2} \sqrt{C_{1}\left(C_{\Pi}^{2 k} N^{2 k(2-m)}+N^{2-2 m}\right)+N^{2(2-m)}}+C_{2} \delta t,
\end{aligned}
$$

where $C_{2}:=C \exp (2)$ is independent of $\delta t$ and $C_{0}$. then $\delta t<\frac{1}{2 C}$ can be guaranteed by

$$
\delta t<\frac{1}{C_{2}} .
$$


Thanks to 4.30, 4.39, 4.41, 4.44 and $m \geq 3$, we have

$$
\begin{aligned}
\left|1-\xi^{n+1}\right| & \leq C\left(\left|E\left[\boldsymbol{u}\left(t^{n+1}\right)\right]-E\left(\overline{\boldsymbol{u}}^{n+1}\right)\right|+\left|s^{n+1}\right|\right) \\
& \leq C\left(\left\|\nabla \overline{\boldsymbol{e}}^{n+1}\right\|+\left|s^{n+1}\right|\right) \\
& \leq C \sqrt{C_{1}\left(1+C_{0}^{2 k}\right) \delta t^{2 k}+C_{1}\left(C_{\Pi}^{2 k} N^{2 k(2-m)}+N^{2-2 m}\right)+C N^{2(1-m)}} \\
& +C_{2} \delta t^{k} \sqrt{C_{1}\left(1+C_{0}^{2 k}\right)}+C_{2} \sqrt{C_{1}\left(C_{\Pi}^{2 k} N^{2 k(2-m)}+N^{2-2 m}\right)+N^{2(2-m)}}+C_{2} \delta t \\
& \leq C_{3} \delta t\left(\sqrt{1+C_{0}^{2 k}} \delta t^{k-1}+1\right)+C_{3} N^{2-m}\left(\sqrt{C_{\Pi}^{2 k} N^{(4-2 m)(k-1)}+N^{-2}+1}\right),
\end{aligned}
$$

where the constant $C_{3}$ is independent of $C_{0}, C_{\Pi}, \delta t$ and $N$. Without loss of generality, we assume $C_{3}>\max \left\{C_{1}, C_{2}, 1\right\}$ to simplify the proof below.

For the cases $k=2,3,4,5$, we choose $C_{0}=2 C_{3}$ and $\delta t \leq \frac{1}{1+C_{0}^{k}}$ to obtain

$$
C_{3}\left(\sqrt{1+C_{0}^{2 k}} \delta t^{k-1}+1\right) \leq C_{3}\left[\left(1+C_{0}^{k}\right) \delta t+1\right] \leq 2 C_{3}=C_{0},
$$

and since $m \geq 3$, we can choose $C_{\Pi}=3 C_{3}$ and $N \geq C_{\Pi}^{k}+1$ to obtain

$$
C_{3}\left(\sqrt{C_{\Pi}^{2 k} N^{(4-2 m)(k-1)}+N^{-2}+1}\right) \leq C_{3}\left[C_{\Pi}^{k} N^{2-m}+2\right] \leq 3 C_{3}=C_{\Pi} .
$$

For the case $k=1$, since $\eta_{1}^{n+1}=1-\left(1-\xi^{n+1}\right)^{2}$, we choose $C_{0}=2 C_{3}$ and $\delta t \leq \frac{1}{1+C_{0}^{2}}$ so that

$$
C_{3}\left(\sqrt{1+C_{0}^{4}} \delta t+1\right) \leq C_{3}\left[\left(1+C_{0}^{2}\right) \delta t+1\right] \leq 2 C_{3}=C_{0}
$$

and since $m \geq 3$, we choose $C_{\Pi}=3 C_{3}$ and $N \geq C_{\Pi}^{2}+1$ to obtain

$$
C_{3}\left(\sqrt{C_{\Pi}^{4} N^{(4-2 m)}+N^{-2}+1}\right) \leq C_{3}\left[C_{\Pi}^{2} N^{2-m}+2\right] \leq 3 C_{3}=C_{\Pi} .
$$

To summarize, combining the above with 4.46, we derive from 4.46 that

$$
\left|1-\xi^{n+1}\right| \leq C_{0} \delta t+C_{\Pi} N^{2-m}
$$

under the conditions

$$
\delta t \leq \frac{1}{1+2^{k+2} C_{0}^{k+1}}, \quad, N \geq 2^{k+2} C_{\Pi}^{k+1}+1 \quad 1 \leq k \leq 5 .
$$

Note that the above implies (4.7), and with $C_{3}>\max \left\{C_{1}, C_{2}, 1\right\}$, it also implies 4.45). The induction process for 4.3 is complete.

We derive from $3.14 \mathrm{~d}$ and 4.33 that

$$
\left\|\boldsymbol{u}_{N}^{n+1}-\overline{\boldsymbol{u}}_{N}^{n+1}\right\|_{1}^{2} \leq\left|\eta_{k}^{n+1}-1\right|^{2}\left\|\overline{\boldsymbol{u}}_{N}^{n+1}\right\|_{1}^{2} \leq\left|\eta_{k}^{n+1}-1\right|^{2} C,
$$

and

$$
\begin{aligned}
\delta t \sum_{q=0}^{n}\left\|\boldsymbol{u}_{N}^{q+1}-\overline{\boldsymbol{u}}_{N}^{q+1}\right\|_{2}^{2} & \leq \delta t \sum_{q=0}^{n}\left|\eta_{k}^{q+1}-1\right|^{2}\left\|\overline{\boldsymbol{u}}_{N}^{q+1}\right\|_{2}^{2} \\
& \leq \max _{q}\left|\eta_{k}^{q+1}-1\right|^{2} \delta t \sum_{q=0}^{n}\left\|\overline{\boldsymbol{u}}_{N}^{q+1}\right\|_{2}^{2} \\
& \leq \max _{q}\left|\eta_{k}^{q+1}-1\right|^{2} C .
\end{aligned}
$$

On the other hand, we derive from (4.3) that

$$
\begin{array}{lll}
\left|\eta_{1}^{q+1}-1\right| \leq 2^{2} C_{0}^{2} \delta t^{2}+2^{2} C_{\Pi}^{2} N^{2(2-m)}, & \forall q \leq n & k=1, \\
\left|\eta_{k}^{q+1}-1\right| \leq 2^{k} C_{0}^{k} \delta t^{k}+2^{k} C_{\Pi}^{k} N^{k(2-m)}, & \forall q \leq n & k=2,3,4,5 .
\end{array}
$$


Therefore, we derive from 4.31, 4.32, 4.51, 4.52, 4.53) and the triangle inequality that

$$
\left\|\boldsymbol{e}^{n+1}\right\|_{1}^{2} \leq\left\|\overline{\boldsymbol{e}}^{n+1}\right\|_{1}^{2}+\left\|\boldsymbol{u}_{N}^{n+1}-\overline{\boldsymbol{u}}_{N}^{n+1}\right\|_{1}^{2},
$$

and

$$
\left\|\boldsymbol{e}^{q+1}\right\|_{2}^{2} \leq\left\|\overline{\boldsymbol{e}}^{q+1}\right\|_{2}^{2}+\left\|\boldsymbol{u}_{N}^{q+1}-\overline{\boldsymbol{u}}_{N}^{q+1}\right\|_{2}^{2}, \quad \forall q \leq n,
$$

under the condition (4.50) on $\delta t$ and $N$. The proof is now complete since we already proved 4.31) and 4.32 .

Using exactly the same procedure above without the spatial discretization, we can prove the following result for the semi-discrete schemes (3.6).

Corollary 1. Let $d=2, T>0, \boldsymbol{u}_{0} \in \boldsymbol{V} \cap \boldsymbol{H}_{p}^{2}$ and $\boldsymbol{u}$ be the solution of (1.1). We assume that $\overline{\boldsymbol{u}}^{i}$ and $\boldsymbol{u}^{i}(i=1, \cdots, k-1)$ are computed with a proper initialization procedure such that for $(i=1, \cdots, k-1)$,

$$
\left\|\overline{\boldsymbol{u}}^{i}-\boldsymbol{u}\left(t_{i}\right)\right\|_{1},\left\|\boldsymbol{u}^{i}-\boldsymbol{u}\left(t_{i}\right)\right\|_{1}=O\left(\delta t^{k}\right) ; \quad\left\|\overline{\boldsymbol{u}}^{i}-\boldsymbol{u}\left(t_{i}\right)\right\|_{2},\left\|\boldsymbol{u}^{i}-\boldsymbol{u}\left(t_{i}\right)\right\|_{2}=O\left(\delta t^{k}\right), \quad i=1,2,3,4,5 .
$$

Let $\overline{\boldsymbol{u}}^{n+1}$ and $\boldsymbol{u}^{n+1}$ be computed with the $k$-th order scheme $(3.6)(1 \leq k \leq 5)$, and

$$
\eta_{1}^{n+1}=1-\left(1-\xi^{n+1}\right)^{2}, \quad \eta_{k}^{n+1}=1-\left(1-\xi^{n+1}\right)^{k}(k=2,3,4,5) .
$$

Then for $n+1 \leq T / \delta$ t and $\delta t \leq \frac{1}{1+2^{k+2} C_{0}^{k+1}}$, we have

$$
\left\|\overline{\boldsymbol{u}}^{n}-\boldsymbol{u}\left(\cdot, t^{n}\right)\right\|_{1}^{2},\left\|\boldsymbol{u}^{n}-\boldsymbol{u}\left(\cdot, t^{n}\right)\right\|_{1}^{2} \leq C \delta t^{2 k}
$$

and

$$
\delta t \sum_{q=0}^{n}\left\|\overline{\boldsymbol{u}}^{q+1}-\boldsymbol{u}\left(\cdot, t^{q+1}\right)\right\|_{2}^{2}, \delta t \sum_{q=0}^{n}\left\|\boldsymbol{u}^{q+1}-\boldsymbol{u}\left(\cdot, t^{q+1}\right)\right\|_{2}^{2} \leq C \delta t^{2 k} .
$$

where the constants $C_{0}$ and $C$ are dependent on $T, \Omega$, the $k \times k$ matrix $G=\left(g_{i j}\right)$ in Lemma 4 and the exact solution $\boldsymbol{u}$, but are independent of $\delta t$.

4.3. Error analysis for the velocity in 3D. In the three-dimensional case, it is no longer possible to obtain the global estimates (4.9), 4.10) and (4.11) as in the two-dimensional case. Instead, we shall derive local estimates in analogy to the local existence of strong solution for the 3-D Navier-Stokes equations.

Theorem 3. Let $d=3, T>0, \boldsymbol{u}_{0} \in \boldsymbol{V} \cap \boldsymbol{H}_{p}^{m}$ with $m \geq 3$. We assume that (1.1) admits a unique strong solution $\boldsymbol{u}$ in $C\left([0, T] ; \boldsymbol{H}_{p}^{1}\right) \cap L^{2}\left(0, T ; \boldsymbol{H}_{p}^{2}\right)$. We assume 4.1] as in Theorem 2, and let $\overline{\boldsymbol{u}}_{N}^{n+1}$ and $\boldsymbol{u}_{N}^{n+1}$ be computed using the $k$ th-order scheme $(3.14)(1 \leq k \leq 5)$, and

$$
\eta_{1}^{n+1}=1-\left(1-\xi^{n+1}\right)^{2}, \quad \eta_{k}^{n+1}=1-\left(1-\xi^{n+1}\right)^{k}(k=2,3,4,5) .
$$

Then, there exits $T_{*}>0$ such that for $0<T<T_{*}, n+1 \leq T / \delta$ t and $\delta t \leq \frac{1}{1+2^{k+2} C_{0}^{k+1}}, N \geq$ $2^{k+2} C_{\Pi}^{k+1}+1$, we have

$$
\left\|\overline{\boldsymbol{u}}_{N}^{n}-\boldsymbol{u}\left(\cdot, t^{n}\right)\right\|_{1}^{2},\left\|\boldsymbol{u}_{N}^{n}-\boldsymbol{u}\left(\cdot, t^{n}\right)\right\|_{1}^{2} \leq C \delta t^{2 k}+C N^{2(1-m)},
$$

and

$$
\delta t \sum_{q=0}^{n}\left\|\overline{\boldsymbol{u}}_{N}^{q+1}-\boldsymbol{u}\left(\cdot, t^{q+1}\right)\right\|_{2}^{2}, \delta t \sum_{q=0}^{n}\left\|\boldsymbol{u}_{N}^{q+1}-\boldsymbol{u}\left(\cdot, t^{q+1}\right)\right\|_{2}^{2} \leq C \delta t^{2 k}+C N^{2(2-m)},
$$

where the constants $C_{0}, C_{\Pi}, C$ are dependent on $T, \Omega$, the $k \times k$ matrix $G=\left(g_{i j}\right)$ in Lemma 4 and the exact solution $\boldsymbol{u}$, but are independent of $\delta t$ and $N$.

Proof. The proof follows essentially the same procedure as the proof for Theorem 2 . However, since we only has the weak version of the stability in Theorem $\mathbf{1}$ and 2.7 is not valid when $d=3$, we can only get a local version of 4.9 ) and 4.10 . To simplify the presentation, we shall only point out below the main differences with the proof for Theorem 2 . 
With $\boldsymbol{u}_{0} \in \boldsymbol{H}_{p}^{m}$ and the existence of a unique strong solution $\boldsymbol{u}$ in $C\left([0, T] ; \boldsymbol{H}_{p}^{1}\right) \cap L^{2}\left(0, T ; \boldsymbol{H}_{p}^{2}\right)$, regularity results in [33, 11] imply that 4.2 is also valid in the three-dimensional case.

In Step 1, we still assume (4.4) holds and choose $\delta t$ and $N$ satisfies 4.7). Let $v_{N}=-\Delta \overline{\boldsymbol{u}}^{n+1}+$ $\tau_{k} \Delta \overline{\boldsymbol{u}}^{n}$ in $3.14 \mathrm{a}$, it follows from Lemma 4 that

$$
\begin{aligned}
\sum_{i, j=1}^{k} g_{i j}( & \left.\nabla \overline{\boldsymbol{u}}_{N}^{q+1+i-k}, \nabla \overline{\boldsymbol{u}}_{N}^{q+1+j-k}\right)-\sum_{i, j=1}^{k} g_{i j}\left(\nabla \overline{\boldsymbol{u}}_{N}^{q+i-k}, \nabla \overline{\boldsymbol{u}}_{N}^{q+j-k}\right) \\
& +\left\|\sum_{i=0}^{k} \delta_{i} \nabla \overline{\boldsymbol{u}}_{N}^{q+1+i-k}\right\|^{2}+\delta t \nu\left\|\Delta \overline{\boldsymbol{u}}_{N}^{q+1}\right\|^{2} \\
& =\delta t \nu\left(\Delta \overline{\boldsymbol{u}}_{N}^{q+1}, \tau_{k} \Delta \overline{\boldsymbol{u}}_{N}^{q}\right)+\delta t\left(\mathbf{A}\left(\left(B_{k}\left(\boldsymbol{u}_{N}^{q}\right) \cdot \nabla\right) B_{k}\left(\boldsymbol{u}_{N}^{q}\right)\right),-\Delta \overline{\boldsymbol{u}}_{N}^{q+1}+\tau_{k} \Delta \overline{\boldsymbol{u}}_{N}^{q}\right) .
\end{aligned}
$$

We now bound the right hand side of 4.56 . Note that 4.7 implies

$$
\frac{1}{2}<1-\left(\frac{\delta t^{k-1}}{4}+\frac{N^{k(2-m)+1}}{4}\right) \leq\left|\eta_{k}^{q}\right| \leq 1+\frac{\delta t^{k-1}}{4}+\frac{N^{k(2-m)+1}}{4}<2, \forall q \leq n .
$$

First, we have

$$
\left|\delta t \nu\left(\Delta \overline{\boldsymbol{u}}_{N}^{q+1}, \tau_{k} \Delta \overline{\boldsymbol{u}}_{N}^{q}\right)\right| \leq \delta t \frac{\nu}{2}\left\|\Delta \overline{\boldsymbol{u}}_{N}^{q+1}\right\|^{2}+\delta t \frac{\nu \tau_{k}}{2}\left\|\Delta \overline{\boldsymbol{u}}_{N}^{q}\right\|^{2} .
$$

Next, it follows from $(2.8)$ that

$$
\begin{aligned}
& \left|\left(\mathbf{A}\left(\left(B_{k}\left(\boldsymbol{u}_{N}^{q}\right) \cdot \nabla\right) B_{k}\left(\boldsymbol{u}_{N}^{q}\right)\right),-\Delta \overline{\boldsymbol{u}}_{N}^{q+1}+\tau_{k} \Delta \overline{\boldsymbol{u}}_{N}^{q}\right)\right| \\
& \quad \leq C\left\|B_{k}\left(\boldsymbol{u}_{N}^{q}\right)\right\|_{1}\left\|B_{k}\left(\nabla \boldsymbol{u}_{N}^{q}\right)\right\|_{1 / 2}\left\|-\Delta \overline{\boldsymbol{u}}_{N}^{q+1}+\tau_{k} \Delta \overline{\boldsymbol{u}}_{N}^{q}\right\| \\
& \quad \leq C\left\|B_{k}\left(\boldsymbol{u}_{N}^{q}\right)\right\|_{1}\left\|B_{k}\left(\boldsymbol{u}_{N}^{q}\right)\right\|_{1}^{1 / 2}\left\|B_{k}\left(\boldsymbol{u}_{N}^{q}\right)\right\|_{2}^{1 / 2}\left\|-\Delta \overline{\boldsymbol{u}}_{N}^{q+1}+\tau_{k} \Delta \overline{\boldsymbol{u}}_{N}^{q}\right\| \\
& \quad \leq C(\varepsilon)\left\|B_{k}\left(\boldsymbol{u}_{N}^{q}\right)\right\|_{1}^{3}\left\|B_{k}\left(\boldsymbol{u}_{N}^{q}\right)\right\|_{2}+\varepsilon\left\|-\Delta \overline{\boldsymbol{u}}_{N}^{q+1}+\tau_{k} \Delta \overline{\boldsymbol{u}}_{N}^{q}\right\|^{2} \\
& \quad \leq C(\varepsilon)\left\|B_{k}\left(\boldsymbol{u}_{N}^{q}\right)\right\|_{1}^{6}+\varepsilon\left\|B_{k}\left(\boldsymbol{u}_{N}^{q}\right)\right\|_{2}^{2}+2 \varepsilon\left\|\Delta \overline{\boldsymbol{u}}_{N}^{q+1}\right\|^{2}+2 \varepsilon\left\|\Delta \overline{\boldsymbol{u}}_{N}^{q}\right\|^{2} .
\end{aligned}
$$

Now, combining (4.56)-(4.58) and noting that $\boldsymbol{u}_{N}^{q}=\eta_{k}^{q} \overline{\boldsymbol{u}}_{N}^{q}$, we find after dropping some unnecessary terms that

$$
\begin{aligned}
\sum_{i, j=1}^{k} g_{i j} & \left(\nabla \overline{\boldsymbol{u}}_{N}^{q+1+i-k}, \nabla \overline{\boldsymbol{u}}_{N}^{q+1+j-k}\right)-\sum_{i, j=1}^{k} g_{i j}\left(\nabla \overline{\boldsymbol{u}}_{N}^{q+i-k}, \nabla \overline{\boldsymbol{u}}_{N}^{q+j-k}\right)+\delta t\left(\frac{\nu}{2}-2 \varepsilon\right)\left\|\Delta \overline{\boldsymbol{u}}_{N}^{q+1}\right\|^{2} \\
\leq & \delta t\left(\frac{\nu \tau_{k}}{2}+2 \varepsilon\right)\left\|\Delta \overline{\boldsymbol{u}}_{N}^{q}\right\|^{2}+\varepsilon \delta t\left\|B_{k}\left(\boldsymbol{u}_{N}^{q}\right)\right\|_{2}^{2}+C(\varepsilon) \delta t\left\|B_{k}\left(\boldsymbol{u}_{N}^{q}\right)\right\|_{1}^{6} \\
\leq & \delta t\left(\frac{\nu \tau_{k}}{2}+2 \varepsilon\right)\left\|\Delta \overline{\boldsymbol{u}}_{N}^{q}\right\|^{2}+2^{2} \varepsilon \delta t\left\|B_{k}\left(\overline{\boldsymbol{u}}_{N}^{q}\right)\right\|_{2}^{2}+2^{6} C(\varepsilon) \delta t\left\|B_{k}\left(\overline{\boldsymbol{u}}_{N}^{q}\right)\right\|_{1}^{6}
\end{aligned}
$$

Taking the sum of 4.59 for $q$ from $k-1$ to $n-1$, noting that $G=\left(g_{i j}\right)$ is a symmetric positive definite matrix with the minimum eigenvalue $\lambda_{G}$ and $\tau_{k}<1$, we can choose $\varepsilon$ small enough such that:

$$
\begin{aligned}
\lambda_{G}\left\|\overline{\boldsymbol{u}}_{N}^{n}\right\|_{1}^{2} & +\frac{\delta t \nu\left(1-\tau_{k}\right)}{4} \sum_{q=0}^{n}\left\|\Delta \overline{\boldsymbol{u}}_{N}^{q}\right\|^{2} \\
& \leq \sum_{i, j=1}^{k} g_{i j}\left(\nabla \overline{\boldsymbol{u}}^{n+i-k}, \nabla \overline{\boldsymbol{u}}^{n+j-k}\right)+\frac{\delta t \nu\left(1-\tau_{k}\right)}{4} \sum_{q=0}^{n}\left\|\Delta \overline{\boldsymbol{u}}_{N}^{q}\right\|^{2} \\
& \leq C \delta t \sum_{q=0}^{n-1}\left\|\overline{\boldsymbol{u}}_{N}^{q}\right\|_{1}^{6}+M_{0},
\end{aligned}
$$

where $M_{0}>0$ is a constant only depends on $\overline{\boldsymbol{u}}_{N}^{0}, \ldots, \overline{\boldsymbol{u}}_{N}^{k}, g_{i j}$. If we define $\phi$ as $\phi(x)=x^{6}$ and let

$$
0<T_{*}<\int_{M_{0}}^{\infty} d z / \phi(z)
$$


then Lemma 5 implies that there exist $C_{*}>0$ independent of $\delta t$ such that

$$
\left\|\overline{\boldsymbol{u}}_{N}^{n}\right\|_{1}^{2}+\delta t \sum_{q=0}^{n}\left\|\Delta \overline{\boldsymbol{u}}_{N}^{q}\right\|^{2} \leq C_{*}, \quad \forall n<T_{*} / \delta t .
$$

With (4.61) holds true, we can then prove (4.54) and (4.55) by following the same procedures in Step 2 and Step 3 in the proof of Theorem 2

Similarly, we can prove the following result for the semi-discrete scheme (3.6).

Corollary 2. Let $d=3, T>0, \boldsymbol{u}_{0} \in \boldsymbol{V} \cap \boldsymbol{H}_{p}^{m}$ with $m \geq 3$. We assume that (1.1) admits a unique strong solution $\boldsymbol{u}$ in $C\left([0, T] ; \boldsymbol{H}_{p}^{1}\right) \cap L^{2}\left(0, T ; \boldsymbol{H}_{p}^{2}\right)$. We assume [4.1] as in Theorem 2, and let $\overline{\boldsymbol{u}}^{n+1}$ and $\boldsymbol{u}^{n+1}$ be computed using the kth-order schemes (3.6), and

$$
\eta_{1}^{n+1}=1-\left(1-\xi^{n+1}\right)^{2}, \quad \eta_{k}^{n+1}=1-\left(1-\xi^{n+1}\right)^{k}(k=2,3,4,5) .
$$

Then, there exits $T_{*}>0$ such that for $0<T<T_{*}, n+1 \leq T / \delta$ t and $\delta t \leq \frac{1}{1+2^{k+2} C_{0}^{k+1}}, N \geq$ $2^{k+2} C_{\Pi}^{k+1}+1$, we have

$$
\left\|\overline{\boldsymbol{u}}^{n}-\boldsymbol{u}\left(\cdot, t^{n}\right)\right\|_{1}^{2},\left\|\boldsymbol{u}^{n}-\boldsymbol{u}\left(\cdot, t^{n}\right)\right\|_{1}^{2} \leq C \delta t^{2 k}
$$

and

$$
\delta t \sum_{q=0}^{n}\left\|\overline{\boldsymbol{u}}^{q+1}-\boldsymbol{u}\left(\cdot, t^{q+1}\right)\right\|_{2}^{2}, \delta t \sum_{q=0}^{n}\left\|\boldsymbol{u}^{q+1}-\boldsymbol{u}\left(\cdot, t^{q+1}\right)\right\|_{2}^{2} \leq C \delta t^{2 k},
$$

where $T_{*}$ is defined in 4.60, the constants $C_{0}, C_{\Pi}, C$ are dependent on $T_{*}, \Omega$, the $k \times k$ matrix $G=\left(g_{i j}\right)$ in Lemma 4 and the exact solution $\boldsymbol{u}$, but are independent of $\delta t$.

4.4. Error analysis for the pressure. With the established error estimates for the velocity $\boldsymbol{u}$, the error estimate for the pressure $p$ can be derived directly from (3.7) or 3.15).

We denote

$$
e_{p N}^{n}:=p_{N}^{n}-\Pi_{N} p\left(\cdot, t^{n}\right), e_{p \Pi}^{n}:=\Pi_{N} p\left(\cdot, t^{n}\right)-p\left(\cdot, t^{n}\right), \text { and } e_{p}^{n}=e_{p N}^{n}+e_{p \Pi}^{n}
$$

Theorem 4. Under the same assumptions as in Theorem 2 and Theorem 3 , we have

$$
\left\|p_{N}^{n+1}-p\left(\cdot, t^{n+1}\right)\right\|^{2} \leq\left\{\begin{array}{l}
C \delta t^{2 k}+C N^{2(1-m)}, \forall n \leq T / \delta t, \quad d=2, \\
C \delta t^{2 k}+C N^{2(1-m)}, \forall n \leq T_{*} / \delta t, \quad d=3 .
\end{array}\right.
$$

and

$$
\delta t \sum_{q=0}^{n}\left\|\nabla\left(p_{N}^{n+1}-p\left(\cdot, t^{n+1}\right)\right)\right\|^{2} \leq\left\{\begin{array}{l}
C \delta t^{2 k}+C N^{2(2-m)}, \quad \forall n \leq T / \delta t, \quad d=2 \\
C \delta t^{2 k}+C N^{2(2-m)}, \quad \forall n \leq T_{*} / \delta t, \quad d=3 .
\end{array}\right.
$$

where $p_{N}^{n+1}$ is computed from (3.15), $T_{*}$ is defined in (4.60) and $C$ is a constant independent of $\delta t$ and $N$.

Proof. From 3.15, we can write down the error equation for $p_{N}^{n+1}$ as

$$
\left(\nabla e_{p}^{q+1}, \nabla v_{N}\right)=\left(\boldsymbol{u}_{N}^{q+1} \cdot \nabla \boldsymbol{u}_{N}^{q+1}-\boldsymbol{u}\left(t^{q+1}\right) \cdot \nabla \boldsymbol{u}\left(t^{q+1}\right), \nabla v_{N}\right), \forall v_{N} \in S_{N}, \forall q+1 \leq n .
$$

To prove 4.62, we set $v_{N}=\Delta^{-1} e_{p N}^{q+1}$ in 4.64 to obtain

$$
\begin{aligned}
\left\|e_{p N}^{q+1}\right\|^{2} & =\left(\boldsymbol{u}_{N}^{q+1} \cdot \nabla\left[\boldsymbol{u}_{N}^{q+1}-\boldsymbol{u}\left(t^{q+1}\right)\right], \Delta^{-\frac{1}{2}} e_{p N}^{q+1}\right) \\
& -\left(\left[\boldsymbol{u}\left(t^{q+1}\right)-\boldsymbol{u}_{N}^{q+1}\right] \cdot \nabla \boldsymbol{u}\left(t^{q+1}\right), \Delta^{-\frac{1}{2}} e_{p N}^{q+1}\right)
\end{aligned}
$$


We can bound the righthand side of 4.65 by using 2.9 , the stability result Theorem 1 and error analysis for the velocity, namely, we can obtain

$$
\begin{aligned}
\left|\left(\boldsymbol{u}_{N}^{q+1} \cdot \nabla\left[\boldsymbol{u}_{N}^{q+1}-\boldsymbol{u}\left(t^{q+1}\right)\right], \Delta^{-\frac{1}{2}} e_{p N}^{q+1}\right)\right| & \leq C(\varepsilon)\left\|\boldsymbol{u}_{N}^{q+1}\right\|_{1}^{2}\|\| \boldsymbol{e}^{q+1}\left\|_{1}^{2}+\varepsilon\right\| \nabla e_{p N}^{q+1} \|^{2} \\
& \leq C(\varepsilon)\left(\delta t^{2 k}+N^{2(1-m)}\right)+\varepsilon\left\|e_{p N}^{q+1}\right\|^{2}
\end{aligned}
$$

and

$$
\begin{aligned}
\left|-\left(\left[\boldsymbol{u}\left(t^{q+1}\right)-\boldsymbol{u}_{N}^{q+1}\right] \cdot \nabla \boldsymbol{u}\left(t^{q+1}\right), \Delta^{-\frac{1}{2}} e_{p N}^{q+1}\right)\right| & \leq C(\varepsilon)\left\|\boldsymbol{u}\left(t^{q+1}\right)\right\|_{1}^{2}\|\| \boldsymbol{e}^{q+1}\left\|_{1}^{2}+\varepsilon\right\| \nabla e_{p N}^{q+1} \|^{2} \\
& \leq C(\varepsilon)\left(\delta t^{2 k}+N^{2(1-m)}\right)+\varepsilon\left\|e_{p N}^{q+1}\right\|^{2}
\end{aligned}
$$

Combining 4.65-4.67) with $\varepsilon=\frac{1}{4}$ we obtain

$$
\left\|e_{p N}^{q+1}\right\|^{2} \leq C \delta t^{2 k}+C N^{2(1-m)}, \quad \forall q \leq n .
$$

To prove 4.63, we set $v_{N}=e_{p N}^{q+1}$ in 4.64 to obtain

$$
\begin{aligned}
\left\|\nabla e_{p N}^{q+1}\right\|^{2} & =\left(\boldsymbol{u}_{N}^{q+1} \cdot \nabla\left[\boldsymbol{u}_{N}^{q+1}-\boldsymbol{u}\left(t^{q+1}\right)\right], \nabla e_{p N}^{q+1}\right) \\
& -\left(\left[\boldsymbol{u}\left(t^{q+1}\right)-\boldsymbol{u}_{N}^{q+1}\right] \cdot \nabla \boldsymbol{u}\left(t^{q+1}\right), \nabla e_{p N}^{q+1}\right)
\end{aligned}
$$

Again, we can bound the righthand side of 4.69 in a similar fashion as in 4.66)-(4.67), namely, we can obtain

$$
\begin{aligned}
\left|\left(\boldsymbol{u}_{N}^{q+1} \cdot \nabla\left[\boldsymbol{u}_{N}^{q+1}-\boldsymbol{u}\left(t^{q+1}\right)\right], \nabla e_{p N}^{q+1}\right)\right| & \leq C(\varepsilon)\left\|\boldsymbol{u}_{N}^{q+1}\right\|_{1}^{2}\|\| \boldsymbol{e}^{q+1}\left\|_{2}^{2}+\varepsilon\right\| \nabla e_{p N}^{q+1} \|^{2} \\
& \leq C(\varepsilon)\left\|\boldsymbol{e}^{q+1}\right\|_{2}^{2}+\varepsilon\left\|\nabla e_{p N}^{q+1}\right\|^{2}
\end{aligned}
$$

and

$$
\begin{aligned}
\left|-\left(\left[\boldsymbol{u}\left(t^{q+1}\right)-\boldsymbol{u}_{N}^{q+1}\right] \cdot \nabla \boldsymbol{u}\left(t^{q+1}\right), \nabla e_{p N}^{q+1}\right)\right| & \leq C(\varepsilon)\left\|\boldsymbol{u}\left(t^{q+1}\right)\right\|_{2}^{2}\|\| \boldsymbol{e}^{q+1}\left\|_{1}^{2}+\varepsilon\right\| \nabla e_{p N}^{q+1} \|^{2} \\
& \leq C(\varepsilon)\left(\delta t^{2 k}+N^{2(1-m)}\right)+\varepsilon\left\|\nabla e_{p N}^{q+1}\right\|^{2}
\end{aligned}
$$

Combining 4.69-4.71 with $\varepsilon=\frac{1}{4}$, we obtain

$$
\left\|\nabla e_{p N}^{q+1}\right\|^{2} \leq C\left\|\boldsymbol{e}^{q+1}\right\|_{2}^{2}+C \delta t^{2 k}+C N^{2(1-m)}, \quad \forall q \leq n .
$$

Taking the sum of 4.25 for $q$ from 0 to $n$ and multiplying $\delta t$ on both sides, we arrive at

$$
\delta t \sum_{q=0}^{n}\left\|\nabla e_{p N}^{q+1}\right\|^{2} \leq C \delta t \sum_{q=0}^{n}\left\|\boldsymbol{e}^{q+1}\right\|_{2}^{2}+C \delta t^{2 k}+C N^{2(1-m)} .
$$

Now, with the estimates on $\left\|\boldsymbol{e}^{n}\right\|_{2}^{2}$ in Theorem 2 or Theorem 3 (4.73) leads to

$$
\delta t \sum_{q=0}^{n}\left\|\nabla e_{p N}^{q+1}\right\|^{2} \leq C \delta t^{2 k}+C N^{2(2-m)} .
$$

Finally, we can obtain 4.62 and 4.63 from 4.68, 4.74 and

$$
\left\|\nabla e_{p \Pi}^{q}\right\|^{2} \leq C N^{2(1-m)} \text {. }
$$

Similarly, we can derive the following results for the semi-discrete scheme (3.6).

Corollary 3. Under the same assumptions as in Corollary $\mathbf{1}$ and Corollary 2, we have

$$
\left\|p^{n+1}-p\left(\cdot, t^{n+1}\right)\right\|^{2} \leq\left\{\begin{array}{l}
C \delta t^{2 k}, \forall n \leq T / \delta t, \quad d=2, \\
C \delta t^{2 k}, \quad \forall n \leq T_{*} / \delta t, \quad d=3 .
\end{array}\right.
$$


and

$$
\delta t \sum_{q=0}^{n}\left\|\nabla\left(p^{q+1}-p\left(\cdot, t^{n+1}\right)\right)\right\|^{2} \leq\left\{\begin{array}{l}
C \delta t^{2 k}, \forall n \leq T / \delta t, \quad d=2, \\
C \delta t^{2 k}, \forall n \leq T_{*} / \delta t, \quad d=3 .
\end{array}\right.
$$

where $p^{n+1}$ is computed from (3.7), $T_{*}$ is defined in 4.60 and $C$ is a constant independent of $\delta t$.

\section{CONCluding REMARKS}

We considered numerical approximation of the incompressible Navier-Stokes equations with periodic boundary conditions for which the pressure can be explicitly eliminated, allowing us to construct very efficient IMEX type schemes using Fourier-Galerkin approximation in space. Our high-order semi-discrete-in-time and fully discrete IMEX schemes are based on a scalar auxiliary variable (SAV) approach which enables us to derive uniform bounds for the numerical solution without any restriction on time step size. We also take advantage of an additional energy dissipation law (2.6), which is only valid for the two-dimensional Navier-Stokes equations with periodic boundary conditions, leading to a uniform bound in $H^{1}$-norm, instead of the usual $L^{2}$-norm. By using these uniform bounds and a delicate induction process, we derived global error estimates in $l^{\infty}\left(0, T ; H^{1}\right) \cap l^{2}\left(0, T ; H^{2}\right)$ in the two dimensional case as well as local error estimates in $l^{\infty}\left(0, T ; H^{1}\right) \cap l^{2}\left(0, T ; H^{2}\right)$ in the three dimensional case for our semi-discrete-in-time and fully discrete IMEX schemes up to fifth-order. We also validated our schemes with manufactured exact solutions and with the double shear layer problem. Our numerical results for the double shear layer problem indicate that the SAV approach can effectively prevent numerical solution from blowing up, and that higher-order schemes are preferable for flows with complex structures such as the double shear layer problem with thin layers.

To the best of our knowledge, our numerical schemes are the first unconditionally stable highorder IMEX type schemes for Navier-Stokes equations without any restriction on time step size, and our error estimates are the first for any IMEX type scheme for the Navier-Stokes equations in the three-dimensional case.

While the stability results can be extended to similar schemes for the Navier-Stokes equations with non-periodic boundary conditions, it is non trivial to carry out the corresponding error analysis which will be left as a subject of future endeavor.

\section{REFERENCES}

[1] Robert A Adams and John JF Fournier. Sobolev spaces. Elsevier, 2003.

[2] Garth A Baker, Vassilios A Dougalis, and Ohannes A Karakashian. On a higher order accurate fully discrete galerkin approximation to the navier-stokes equations. Mathematics of Computation, 39(160):339-375, 1982.

[3] John B Bell, Phillip Colella, and Harland M Glaz. A second-order projection method for the incompressible navier-stokes equations. Journal of Computational Physics, 85(2):257-283, 1989.

[4] David L Brown. Performance of under-resolved two-dimensional incompressible flow simulations. Journal of Computational Physics, 122(1):165-183, 1995.

[5] Kelong Cheng and Cheng Wang. Long time stability of high order multistep numerical schemes for two-dimensional incompressible Navier-Stokes equations. SIAM J. Numer. Anal., 54(5):3123-3144, 2016.

[6] Javier de Frutos, Bosco García-Archilla, and Julia Novo. Postprocessing finite-element methods for the NavierStokes equations: the fully discrete case. SIAM J. Numer. Anal., 47(1):596-621, 2008/09.

[7] Michel O Deville, Paul F Fischer, Paul F Fischer, EH Mund, et al. High-order methods for incompressible fluid flow. Number 9. Cambridge university press, 2002.

[8] Yana Di, Ruo Li, Tao Tang, and Pingwen Zhang. Moving mesh finite element methods for the incompressible navier-stokes equations. SIAM Journal on Scientific Computing, 26(3):1036-1056, 2005.

[9] Weinan E. Convergence of Fourier methods for the Navier-Stokes equations. SIAM J. Numer. Anal., 30(3):650$674,1993$.

[10] Weinan E and Jian-Guo Liu. Projection method i: convergence and numerical boundary layers. SIAM journal on numerical analysis, pages 1017-1057, 1995.

[11] Ciprian Foias and Roger Temam. Gevrey class regularity for the solutions of the navier-stokes equations. Journal of Functional Analysis, 87(2):359-369, 1989.

[12] Vivette Girault and P-A Raviart. Finite element approximation of the navier-stokes equations. Lecture Notes in Mathematics, Berlin Springer Verlag, 749, 1979. 
[13] Roland Glowinski. Finite element methods for incompressible viscous flow. Handbook of numerical analysis, 9:31176, 2003.

[14] Sigal Gottlieb, Florentina Tone, Cheng Wang, Xiaoming Wang, and Djoko Wirosoetisno. Long time stability of a classical efficient scheme for two-dimensional navier-stokes equations. SIAM Journal on Numerical Analysis, 50(1):126-150, 2012.

[15] Jean-Luc Guermond, Peter Minev, and Jie Shen. An overview of projection methods for incompressible flows. Computer methods in applied mechanics and engineering, 195(44-47):6011-6045, 2006.

[16] Max D Gunzburger. Finite element methods for viscous incompressible flows: a guide to theory, practice, and algorithms. Elsevier, 2012.

[17] Ole H Hald. Convergence of fourier methods for navier-stokes equations. Journal of Computational Physics, 40(2):305-317, 1981.

[18] Yinnian He and Weiwei Sun. Stability and convergence of the Crank-Nicolson/Adams-Bashforth scheme for the time-dependent Navier-Stokes equations. SIAM J. Numer. Anal., 45(2):837-869, 2007.

[19] Timo Heister, Maxim A Olshanskii, and Leo G Rebholz. Unconditional long-time stability of a velocity-vorticity method for the 2d navier-stokes equations. Numerische Mathematik, 135(1):143-167, 2017.

[20] John G Heywood and Rolf Rannacher. Finite-element approximation of the nonstationary navier-stokes problem. part iv: Error analysis for second-order time discretization. SIAM Journal on Numerical Analysis, 27(2):353-384, 1990.

[21] Fukeng Huang, Jie Shen, and Zhiguo Yang. A highly efficient and accurate new scalar auxiliary variable approach for gradient flows. SIAM Journal on Scientific Computing, 42(4):A2514-A2536, 2020.

[22] Heinz-Otto Kreiss and Joseph Oliger. Stability of the fourier method. SIAM Journal on Numerical Analysis, 16(3):421-433, 1979.

[23] Xiaoli Li and Jie Shen. Error analysis of the sav-mac scheme for the navier-stokes equations. SIAM Journal on Numerical Analysis, 58(5):2465-2491, 2020.

[24] Lianlei Lin, Zhiguo Yang, and Suchuan Dong. Numerical approximation of incompressible navier-stokes equations based on an auxiliary energy variable. Journal of Computational Physics, 388:1-22, 2019.

[25] Jian-Guo Liu, Robert Pego, et al. Stable discretization of magnetohydrodynamics in bounded domains. Communications in Mathematical Sciences, 8(1):235-251, 2010.

[26] Parviz Moin and Krishnan Mahesh. Direct numerical simulation: a tool in turbulence research. Annual review of fluid mechanics, 30(1):539-578, 1998.

[27] Olavi Nevanlinna and F Odeh. Multiplier techniques for linear multistep methods. Numerical Functional Analysis and Optimization, 3(4):377-423, 1981.

[28] Steven A Orszag and GS Patterson Jr. Numerical simulation of three-dimensional homogeneous isotropic turbulence. Physical Review Letters, 28(2):76, 1972.

[29] Roger Peyret. Spectral methods for incompressible viscous flow, volume 148. Springer Science \& Business Media, 2013.

[30] Zhen-Su She, Eric Jackson, and Steven A Orszag. Structure and dynamics of homogeneous turbulence: models and simulations. Proceedings of the Royal Society of London. Series A: Mathematical and Physical Sciences, 434(1890):101-124, 1991.

[31] Jie Shen. Long time stability and convergence for fully discrete nonlinear galerkin methods. Applicable Analysis, 38(4):201-229, 1990.

[32] Jie Shen, Tao Tang, and Li-Lian Wang. Spectral methods: algorithms, analysis and applications, volume 41. Springer Science \& Business Media, 2011.

[33] R. Temam. Navier-Stokes Equations and Nonlinear Functional Analysis. SIAM, Philadelphia, 1983.

[34] R. Temam. Navier-Stokes Equations: Theory and Numerical Analysis. North-Holland, Amsterdam, 1984.

[35] Florentina Tone, Xiaoming Wang, and Djoko Wirosoetisno. Long-time dynamics of 2d double-diffusive convection: analysis and/of numerics. Numerische Mathematik, 130(3):541-566, 2015.

[36] Xiaoming Wang. An efficient second order in time scheme for approximating long time statistical properties of the two dimensional navier-stokes equations. Numerische Mathematik, 121(4):753-779, 2012. 